\title{
Genetic deficiency or pharmacological inhibition of miR-33 protects from kidney fibrosis
}

\begin{abstract}
Nathan L. Price, ${ }^{1,2}$ Verónica Miguel, ${ }^{3}$ Wen Ding, ${ }^{1,2}$ Abhishek K. Singh, ${ }^{1,2}$ Shipra Malik, ${ }^{4}$ Noemi Rotllan, ${ }^{1,2}$ Anna Moshnikova, ${ }^{5}$ Jakub Toczek, ${ }^{1,6,7}$ Caroline Zeiss, ${ }^{2}$ Mehran M. Sadeghi, ${ }^{1,6,7}$ Noemi Arias, ${ }^{8}$ Ángel Baldán, ${ }^{8}$ Oleg A. Andreev, ${ }^{5}$ Diego Rodriguez-Puyol, ${ }^{9}$ Raman Bahal, ${ }^{4}$ Yana K. Reshetnyak, ${ }^{5}$ Yajaira Suárez, ${ }^{1,2}$ Carlos Fernández-Hernando, ${ }^{1,2}$ and Santiago Lamas ${ }^{3}$

'Vascular Biology and Therapeutics Program and 'Integrative Cell Signaling and Neurobiology of Metabolism Program, Department of Comparative Medicine and Department of Pathology, Yale University School of Medicine, New Haven, Connecticut, USA. ${ }^{3}$ Department of Cell Biology and Immunology, Centro de Biología Molecular "Severo Ochoa," Madrid, Spain. ${ }^{4}$ Department of Pharmaceutical Sciences, University of Connecticut, Storrs, Connecticut, USA. ${ }^{5}$ Department of Physics, University of Rhode Island, Kingston, Rhode Island, USA. ${ }^{6}$ Cardiovascular Molecular Imaging Laboratory, Section of Cardiovascular Medicine, and Yale Cardiovascular Research Center, Yale University School of Medicine, New Haven, Connecticut, USA. ${ }^{7}$ Section of Cardiology, Veterans Affairs Connecticut Healthcare System, West Haven, Connecticut, USA. ${ }^{8}$ Edward A. Doisy Department of Biochemistry and Molecular Biology and Center for Cardiovascular Research, Saint Louis University School of Medicine, St. Louis, Missouri, USA. 'Department of Medicine and Medical Specialties, Research Foundation of the University Hospital “Príncipe de Asturias," IRYCIS, Alcalá University, Alcalá de Henares, Madrid, Spain.
\end{abstract}

Previous work has reported the important links between cellular bioenergetics and the development of chronic kidney disease, highlighting the potential for targeting metabolic functions to regulate disease progression. More recently, it has been shown that alterations in fatty acid oxidation (FAO) can have an important impact on the progression of kidney disease. In this work, we demonstrate that loss of miR-33, an important regulator of lipid metabolism, can partially prevent the repression of FAO in fibrotic kidneys and reduce lipid accumulation. These changes were associated with a dramatic reduction in the extent of fibrosis induced in $\mathbf{2}$ mouse models of kidney disease. These effects were not related to changes in circulating leukocytes because bone marrow transplants from miR-33-deficient animals did not have a similar impact on disease progression. Most important, targeted delivery of miR-33 peptide nucleic acid inhibitors to the kidney and other acidic microenvironments was accomplished using $\mathrm{pH}$ low insertion peptides as a carrier. This was effective at both increasing the expression of factors involved in FAO and reducing the development of fibrosis. Together, these findings suggest that miR-33 may be an attractive therapeutic target for the treatment of chronic kidney disease.

Authorship note: NLP and VM contributed equally to this work.

Conflict of interest: OAA and YKR are founders of pHLIP, Inc. They have shares in the company, but the company did not fund any part of the work reported in the paper, which was done in their academic laboratories.

Copyright: (c) 2019, American Society for Clinical Investigation.

Submitted: June 14, 2019

Accepted: October 10, 2019

Published: October 15, 2019

Reference information: JCI Insight 2019;4(22):e131102.

https://doi.org/10.1172/jci.

insight.131102.
Chronic kidney disease (CKD) is a widespread condition affecting over 30 million people in the United States and about $10 \%$ of the population worldwide (1). The progression of this disease results in kidney failure, also known as end-stage renal disease (ESRD). ESRD causes nearly a million deaths each year globally, and over 2 million people are currently receiving treatment. However, it is estimated that this is only a fraction of the people in need of treatment because of the expense and limited availability of currently available therapies (chronic dialysis sessions or organ transplant), leading to over 1 million deaths each year (2).

Fibrosis is the final common pathway and histological manifestation of highly prevalent clinical conditions that lead to $\mathrm{CKD}$, such as hypertension, nephroangiosclerosis, and diabetic nephropathy (DN). It is characterized by the accumulation of collagen, activated myofibroblasts, and inflammatory cells, as well as cell death, epithelial cell dedifferentiation, and loss of vascular supply in the kidney $(3,4)$. Renal tubule cells have high baseline metabolic needs and rely mostly on fatty acids as their primary fuel source to generate energy via mitochondrial oxidative phosphorylation (5). They have high levels of peroxisomal proliferator-activated receptor- $\alpha(\operatorname{PPAR} \alpha)$ and PPAR $\gamma$ coactivator- $1 \alpha(\mathrm{PGC}-1 \alpha)$ and a dense mitochondrial network to support their metabolic and functional needs (6).

\section{Introduction}


Alterations in metabolic function are one of the biggest risk factors for CKD. Elevated blood glucose levels are known to promote kidney dysfunction, and nearly one-third of patients with diabetes develop nephropathy (7). More recently, abnormal renal lipid accumulation has been linked to the development of $\mathrm{CKD}$ and renal fibrosis (8). This has led to the prevailing concept that tubulo-interstitial fibrosis can be mediated or perpetuated by metabolic alterations, whereby reduced fatty acid oxidation (FAO) plays a central role in promoting and prolonging fibrotic responses (9-13). The use of different models of chronic kidney injury leading to renal fibrosis, including unilateral ureteral obstruction (UUO), folic acid-induced nephropathy (FAN), ischemia/reperfusion, or genetically modified models, has allowed researchers to establish that this reduction is associated with decreased expression of numerous FAO-related genes in renal tubular epithelial cells (TECs). Facing those metabolic constraints, TECs that survive injury respond by rearranging their cytoskeleton and acquiring a less differentiated, mesenchymal phenotype (14).

Carnitine palmitoyltransferase 1a (CPT1a) is a mitochondrial enzyme central to the regulation of FAO, through its role in the transport of long chain fatty acids from the cytoplasm into the mitochondria. Reduction in the levels of this enzyme has been linked to an activation of the TGF- $\beta$ axis in the context of inflammatory response, leading to fibrogenesis through the inhibition of PPAR $\alpha$-dependent downstream signaling (11). However, therapies based on the inhibition of TGF- $\beta$ signaling have not yet been proved valuable in human disease. Likewise, the use of fibrates as agonists of the PPAR $\alpha$ family of receptors has been curtailed in the clinic because of side effects involving renal function (15). Thus, it is important to investigate new strategies that may provide a basis for therapeutic targets.

miRNAs are noncoding RNAs whose importance in pathophysiology has steadily grown over the past decade. The ability of a single miRNA to inhibit a large number of mRNA targets can allow miRNAs to promote more robust responses by targeting multiple genes in the same or related pathways or to elicit a broader cellular response by targeting genes in pathways that are generally not related. Although this provides miRNAs the ability to regulate a wide variety of complex cellular responses, it also makes it difficult to determine the specific mechanisms by which miRNAs mediate their effects. Recently, our group was able to demonstrate that disruption of an individual miRNA/target interaction was sufficient to mimic many of the effects of miRNA deficiency in vivo. Although this was the first work demonstrating the capacity of an individual miRNA/target interaction to mediate complex physiological phenotypes, the more moderate phenotype suggests other targets may also have been involved (16). Indeed, in most cases multiple mRNA targets are likely involved in mediating the effects of an miRNA, and to add a new level of complexity, the effects of a given miRNA can vary dramatically among tissues, cell types, and physiological states because of differences in expression and target availability.

Several miRNAs have been related to the regulation of renal responses during fibrosis (17-19). Previous reports demonstrated that downregulation of miR-214 and miR-21, which have increased expression in models of renal damage and are implicated in human renal pathology, is protective against renal fibrosis (20-22). Alternatively, miR-29 expression is suppressed by TGF- $\beta$, promoting collagen expression and renal fibrosis (23). However, the role of miRNAs in the regulation of renal fibrosis is still largely unexplored, and recent work demonstrating the ability of miRNAs to regulate cellular bioenergetics suggests that these novel regulators could constitute a pathogenic link between metabolism and fibrogenesis. We hypothesized that miR-33, a major regulator of lipid metabolism, could represent an attractive candidate in this setting.

The miR-33 family of miRNAs are encoded within the sterol regulatory binding protein (SREBP) genes. SREBP1 and SREBP2 are transcription factors responsible for the regulation of fatty acid and cholesterol biosynthesis. miR-33a and miR-33b are cotranscribed along with their host genes and work in a concerted manner by targeting genes involved in cholesterol efflux $(A B C A 1, A B C G 1)(24,25)$ and FAO (CPT1a , CROT, HADHB) $(26,27)$. miR-33 is highly expressed in human and mouse kidneys (24, 25). Recent work has shown that miR-33 expression is increased in the serum of patients with diabetes and even further elevated in patients with DN. Similarly, rodents with DN had higher levels of miR-33 both in blood and in renal tissue (28). In this work, we utilize miR-33-knockout mice ( $\left.m i R-33^{-/}\right)$to investigate how genetic loss of miR-33 affects the development of kidney fibrosis. Our results indicate that miR-33-deficient animals are protected from kidney fibrosis. This is likely due at least in part to reduced renal lipid accumulation and increased expression of miR-33 target genes involved in FAO. We further demonstrate that selective delivery of miR-33 peptide nucleic acid (PNA) inhibitors to the kidney, and other acidic environments, using a $\underline{\mathrm{pH}}$ low insertion peptide (pHLIP) carrier increased the expression of 
A WT or miR-33-
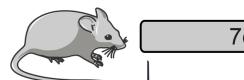

$7 d$

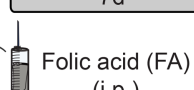

(i.p.)

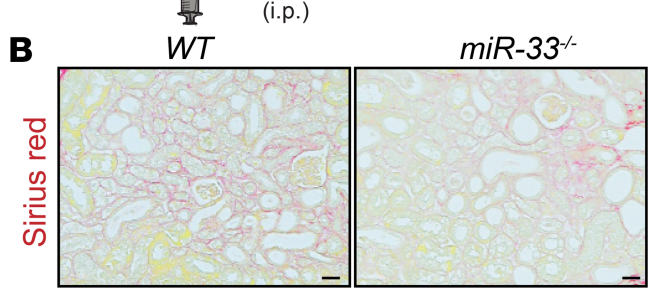

D
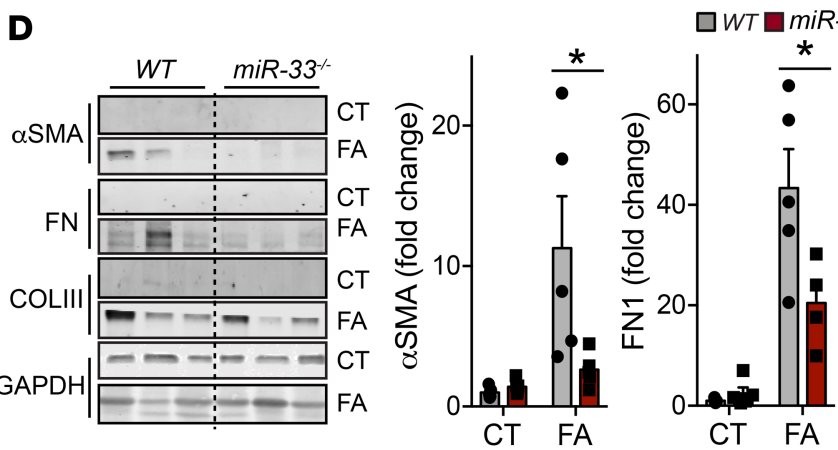

C

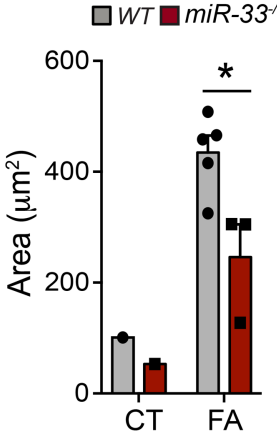

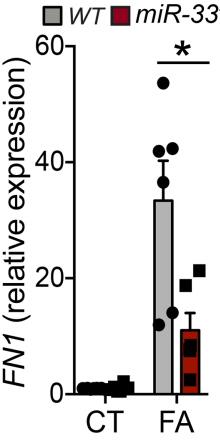

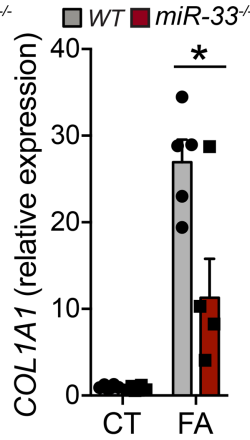

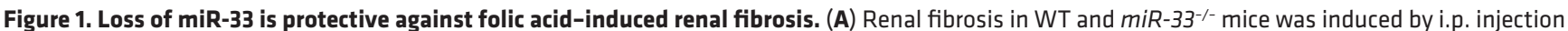
of folic acid (FA) (250 mg/kg body weight) (B). Representative microphotographs from 1 mouse per group and quantification (right) of Picrosirius red

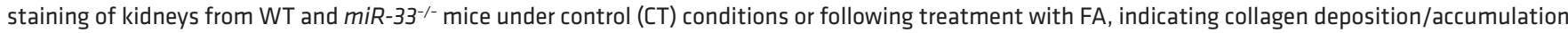
$(n=3-5)$. (C) Quantitative reverse transcription PCR (qRT-PCR) analysis of the expression of fibrosis-associated genes ASMA, FN1, and COL1A1 in kidneys from WT and miR-33-1- mice under CT conditions or following treatment with FA $(n=5-6)$. (D) Representative images and quantification of Western blot analysis of protein expression of fibrosis-associated genes: $\alpha$-SMA, FN1, and COLIII in kidneys from WT and miR-33-1- mice under CT conditions (top) or following treatment with FA (bottom). Relative protein levels were determined by band densitometry and are expressed in AU after correction for loading CT GAPDH $(n=5)$. (E) Quantification of levels of BUN (left) and creatinine (right) in plasma samples of WT and miR-33\%- mice under CT conditions or following treatment with FA $(n=5-7)$. All statistical significance was determined using nonparametric 2 -tailed Mann-Whitney $U$ test. Data represent the mean \pm SEM and ${ }^{*} P<0.05$ comparing $m i R-33^{-/-}$with WT mice under the same conditions. Scale bar: $20 \mu \mathrm{m}$.

enzymes involved in FAO. Similar to what we observed in $m i R-33^{-1-}$ mice, this treatment was effective in reducing kidney fibrosis and inducing factors related to kidney damage. This approach may represent a novel therapeutic avenue for the treatment of kidney disease.

\section{Results}

Loss of miR-33 protects mice against kidney fibrosis. We sought to determine whether miR-33 may play a direct role in promoting the development of kidney dysfunction using 2 common models of kidney fibrosis, FAN and UUO. Seven days after intraperitoneal (i.p.) injection of folic acid (Figure 1A), mice deficient in miR-33 showed a dramatic reduction in the development of kidney fibrosis compared with WT mice. Histological analysis revealed that $m i R-33^{-/-}$mice had reduced accumulation of collagen as visualized by Sirius red staining (Figure 1B). The induction of fibrosis-associated markers ( $\alpha$-smooth muscle actin $[\alpha-S M A]$, fibronectin [FN1], and collagen) in response to folic acid was also reduced in $m i R-33^{-/-}$mice at both the mRNA (Figure 1C) and protein levels (Figure 1D). Furthermore, common parameters indicative of kidney function, blood urea nitrogen (BUN) and creatinine, were increased in animals injected with folic acid while this response

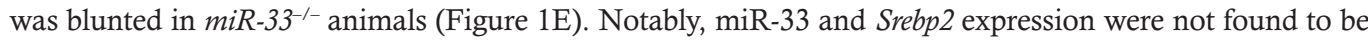
significantly altered in response to FAN (Supplemental Figure 1, A and B; supplemental material available online with this article; https://doi.org/10.1172/jci.insight.131102DS1), suggesting that suppression of basal miR-33 expression was sufficient to protect against folic acid-induced kidney fibrosis.

Similar results were obtained in $m i R-33^{-1-}$ and control animals using an independent model of renal dysfunction, UUO surgery (Figure 2A). In this model UUO surgery is performed only in 1 kidney, leaving the contralateral kidney as a nonfibrotic control. Similar to the findings observed in the FAN model, expression 
A

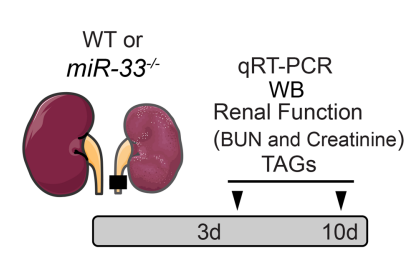

UUO (Unilateral Ureteral Obstruction)

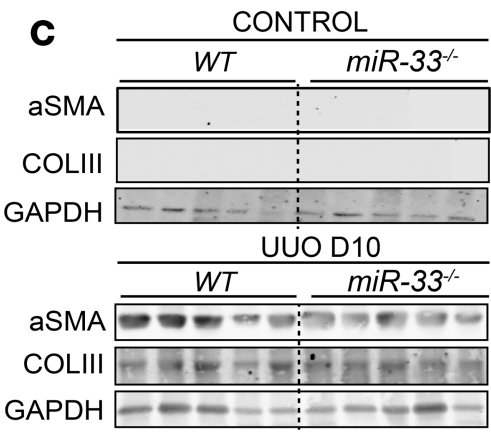

D

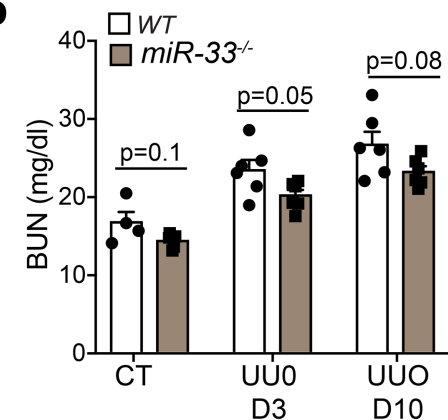

B

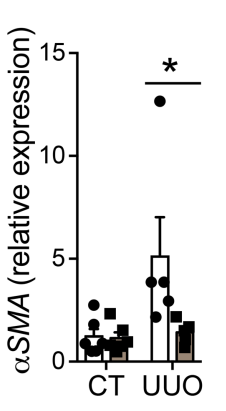

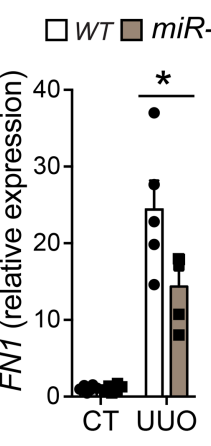

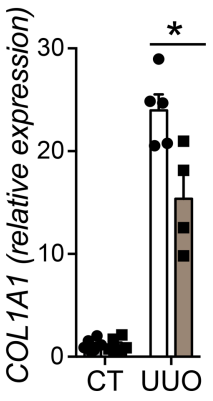

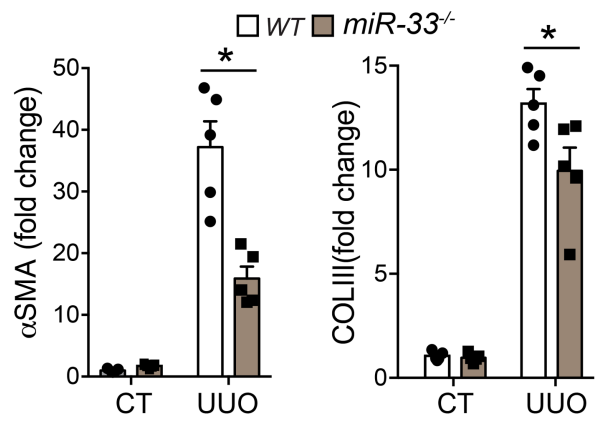

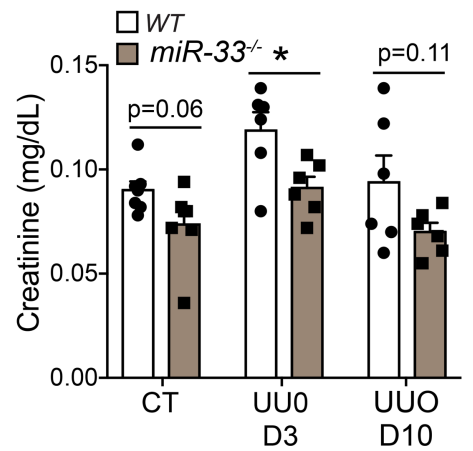

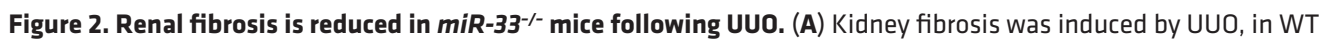
and $m i R-33^{--}$mice. The contralateral kidney was used as a nonfibrotic CT. (B) qRT-PCR analysis of the expression of fibrosis-associated genes ASMA, FN1, and COL1A1 in WT and miR-33\%- mice in CT kidneys or following UUO surgery $(n=4-6)$. (C) Representative images and quantification of Western blot analysis of protein expression of fibrotic fac-

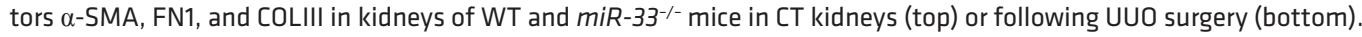
Relative protein levels were determined by band densitometry and are expressed in AU after correction for loading CT GAPDH $(n=5)$. (D) Quantification of levels of BUN (left) and creatinine (right) in plasma samples of WT and $\mathrm{miR}^{-33^{-/-}}$ mice in CT kidneys or after 3 or 10 days of UUO surgery $(n=4-6)$. All statistical significance was determined using nonparametric 2-tailed Mann-Whitney $U$ test. Data represent the mean \pm SEM and ${ }^{*} P<0.05$ comparing miR-33 $3^{-/-}$with WT mice under the same conditions.

of miR-33 and Srebp2 was unaffected by UUO surgical procedure (Supplemental Figure 1, C and D). How-

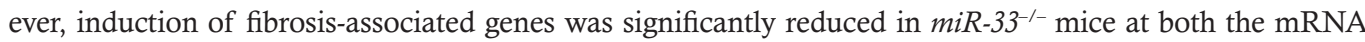
(Figure 2B) and protein levels compared with WT mice (Figure 2C). Despite the presence of a functional contralateral kidney, we also observed a trend toward decreased plasma BUN in $m i R-33^{-1-}$ mice both 3 and 10 days after UUO surgery, along with a significant decrease in creatinine after 3 days of UUO (Figure 2D). Together these findings demonstrate that loss of miR-33 is protective against kidney fibrosis in rodents.

Protection against kidney fibrosis in $\mathrm{miR}-33^{-/-}$mice is not due to changes in circulating macrophages or other hematopoietic cells. Similar to what we observed with fibrotic genes, the induction of mediators of inflammatory response

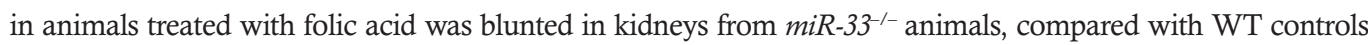
(Figure 3A). Because miR-33 has been shown to play an important role in the development of other chronic inflammatory conditions, such as atherosclerosis, and to regulate macrophage polarization (29), we investigated whether the reduced inflammation and fibrosis of $m i R-33^{--}$mice was due to effects on macrophages or other 

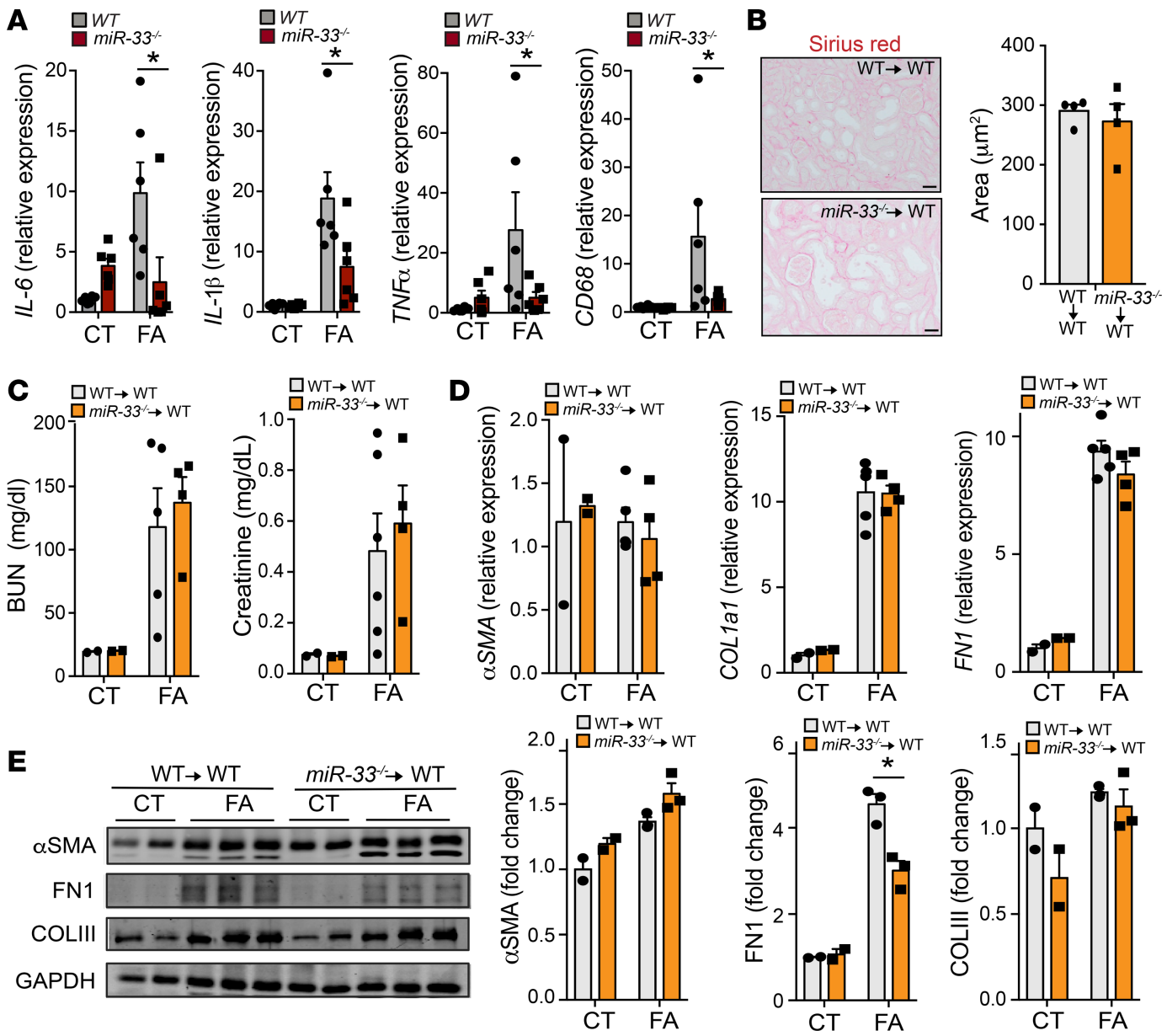

Figure 3. Protection from kidney fibrosis in $\mathrm{miR}^{-33^{-/-}}$mice is not due to effects on circulating leukocytes. (A) qRT-PCR analysis of the expression of inflammatory genes: IL6, IL1B, TNFA, and CD68 in WT and miR-33-- mice under CT conditions or following treatment with FA $(n=6)$. (B) Representative microphotographs from 1 mouse per group and quantification of Picrosirius red staining of kidneys from WT mice reconstituted with BM from WT (WT $\rightarrow$ WT) or miR-33 $3^{-/-}$mice ( $m i R-33^{-/-} \rightarrow$ WT) under CT conditions or following treatment with FA $(n=4)$; quantification is shown at the right. (C) Quantification of levels of BUN (left) and creatinine (right) in plasma samples of WT mice reconstituted with BM from WT and $m i R-33^{-/-}$mice under CT conditions or following treatment with FA $(n=4-6)$. (D) qRT-PCR analysis of the expression

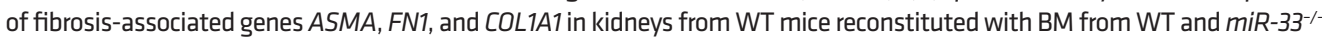
mice under CT conditions or following treatment with FA $(n=4-5)$. (E) Representative images and quantification of Western blot analysis of protein expression of fibrosis-associated genes: $\alpha$-SMA, FN1, and COLIII in kidneys from WT mice reconstituted with $\mathrm{BM}$ from WT and $m i R-33^{-/-}$mice under $\mathrm{CT}$ conditions or following treatment with $\mathrm{FA}$. Relative protein levels were determined by band densitometry and are expressed in AU after correction for loading CT GAPDH $(n=3)$. All statistical significance was determined using nonparametric 2-tailed Mann-Whitney $U$ test. Data represent the mean \pm SEM and ${ }^{*} P<0.05$ comparing WT mice reconstituted with BM from miR-33-1- mice with animals reconstituted with WT $\mathrm{BM}$, under the same conditions. Scale bar: $20 \mu \mathrm{m}$.

inflammatory cells. To this end, we performed bone marrow transplant (BMT) experiments in which lethally

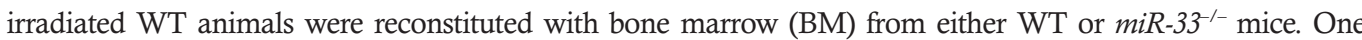
month after BMT, mice were injected with folic acid, and the extent of kidney fibrosis was determined after 7 days. In contrast with our previous observations in $m i R-33^{--}$mice, kidney sections from mice reconstituted with miR-33-- $\mathrm{BM}$ did not show any differences in the amount of Sirius red-positive staining of collagen fibers compared to kidneys from mice reconstituted with BM from WT mice (Figure 3B). Similarly, circulating levels

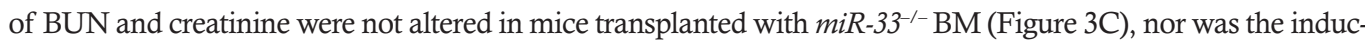
tion of fibrosis-associated genes altered (Figure 3D). Although the protein levels of fibronectin were reduced in $m i R-33^{-1-}$ BMT animals, other fibrotic markers, such as collagen and $\alpha$-SMA, were unaffected (Figure 3E). These findings indicate that the impact of miR-33 on kidney fibrosis is not due to effects on BM-derived macrophages or other precursor hematopoietic cells and thus likely related to a localized effect within the kidney. 
A

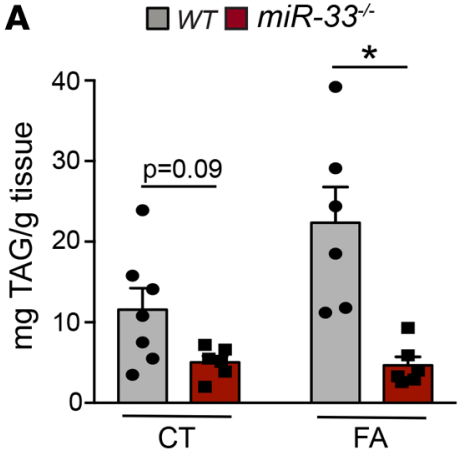

D
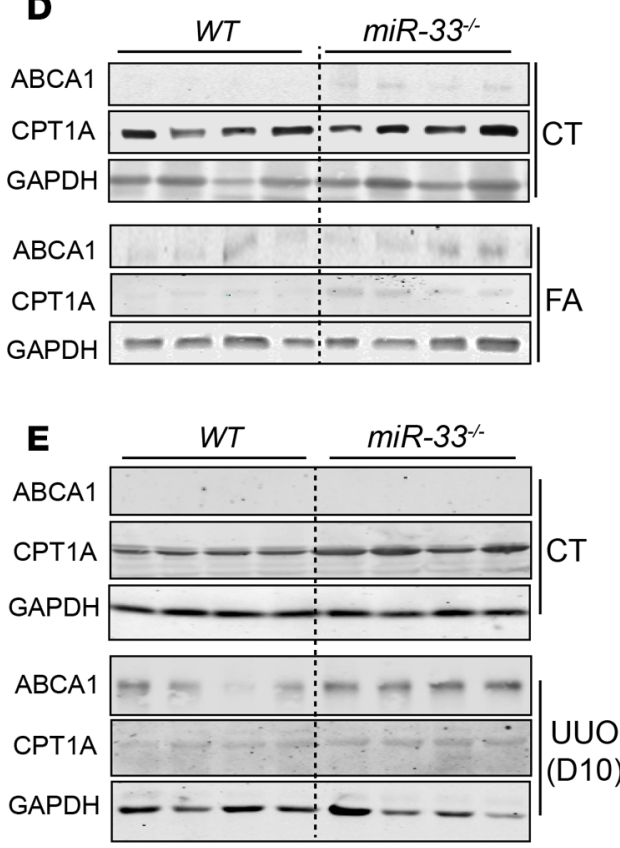
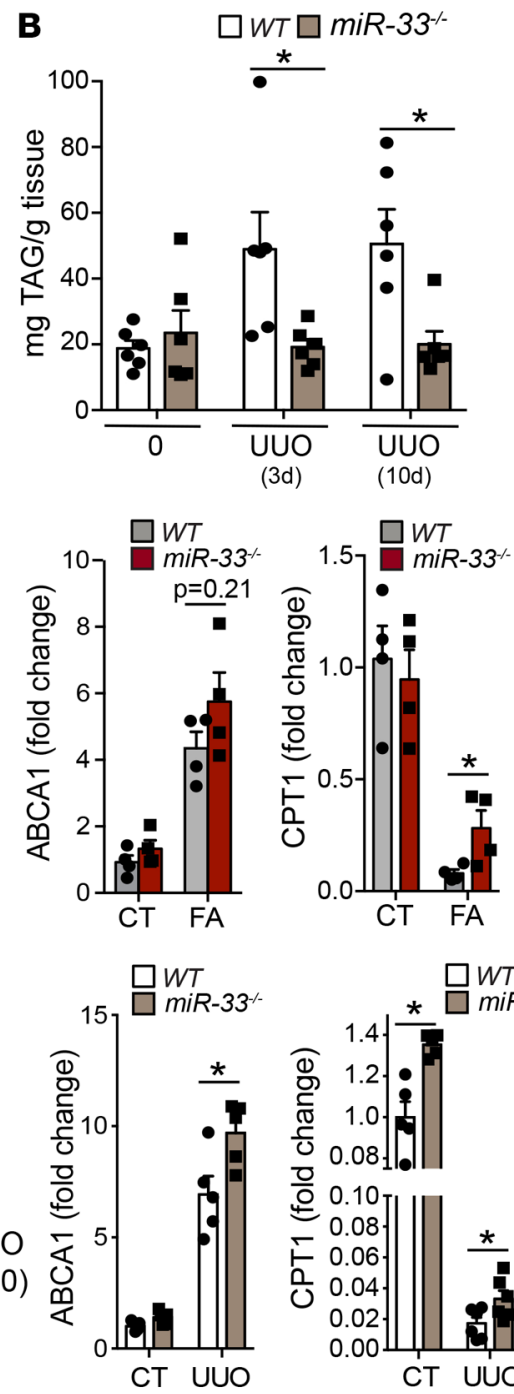

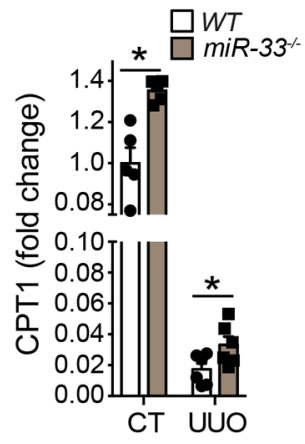

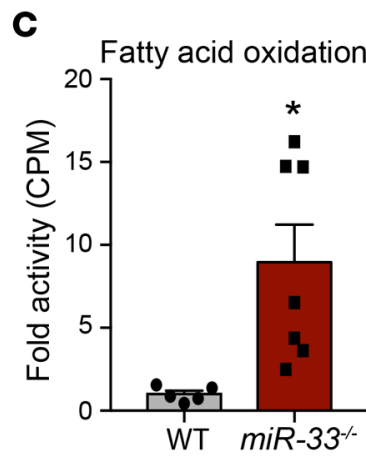

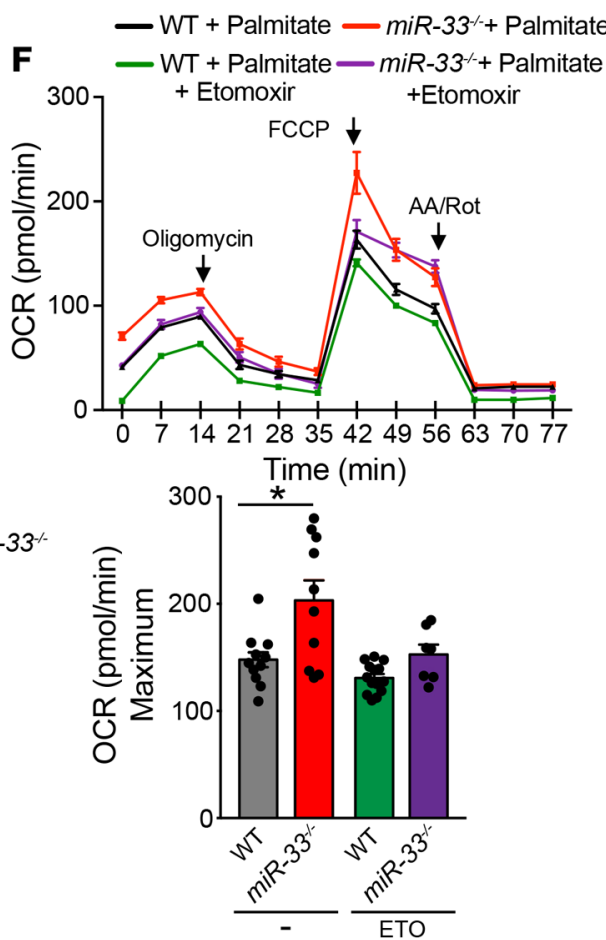

Figure 4. Loss of miR-33 increases FAO and reduces renal lipid accumulation. (A and B) Quantification of triglyceride (TAC) levels in total kidney tissue from WT and miR-33-/- mice under CT conditions or following (A) FA treatment or (B) UUO surgery ( $n=6-7)$. (C) Radiolabeled palmitate-derived $\mathrm{CO}_{2}$ was determined after incubation of ${ }^{14} \mathrm{C}$-palmitate with kidney tissue from WT and miR-33-/- mice $(n=5-7)$. ( $\mathbf{D}$ and $\left.\mathbf{E}\right)$ Representative images and quantification of Western blot analysis of protein expression of miR-33 target genes $A B C A 1$ and CPT1A in the kidneys from WT and miR-33-1- mice under CT conditions or following (D) treatment with FA or (E) UUO surgery. Relative protein levels were determined by band densitometry and are expressed in AU after correction

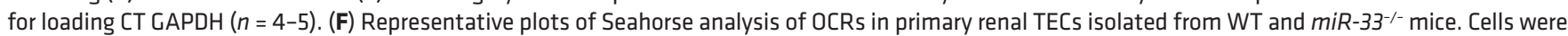
treated with palmitate-BSA FAO substrate. Oligomycin $(1 \mu \mathrm{M})$, FCCP $(3 \mu \mathrm{M})$, and a combination of antimycin A $(1 \mu \mathrm{M})$ and rotenone $(1 \mu \mathrm{M})(\mathrm{AA} / \mathrm{Rot})$ were injected sequentially at the indicated time points. Measurements were performed both under basal conditions and after treatment with the CPT1a inhibitor etomoxir (Eto). Data are represented after normalization by protein amount. Bar graphs show the values of OCR associated with maximal respiratory status (bottom) ( $n=7-14$ replicate wells; results are representative of at least 2 additional experiments with similar results). Statistical significance was determined using nonparametric 2-tailed Mann-Whitney $U$ test (A, B, D, and $\mathbf{E})$, unpaired 2-sided Student's $t$ test (C), and 1-way ANOVA with Bonferroni's correc-

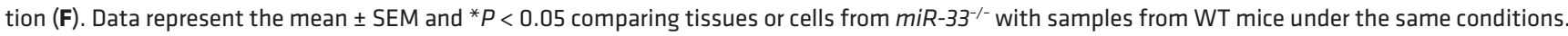

Loss of miR-33 promotes $F A O$ and reduces lipid accumulation in fibrotic kidneys. miR-33 is an important regulator of FAO and cholesterol efflux. Notably, excessive lipid accumulation is known to be involved in kidney dysfunction (30), and impairment in FAO in renal TECs has been associated with enhanced renal fibrosis (14). Hence, we sought to determine whether alterations in lipid metabolism may be responsible for the protective effects of miR33 deficiency. Our data demonstrate that the amount of kidney triglycerides (TAGs) was significantly reduced in

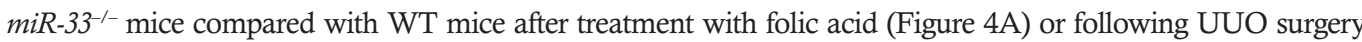

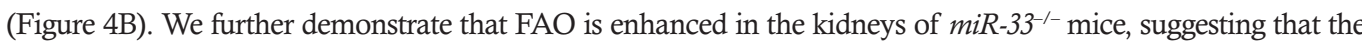
reduced lipid accumulation we observed may be due to increased FAO (Figure 4C). These effects are likely due at least in part to a direct action of miR-33 on CPT1a, a known target of miR-33 and critical regulator of FAO. Western blot analysis demonstrated that CPT1a protein levels were markedly reduced in the kidneys of mice 

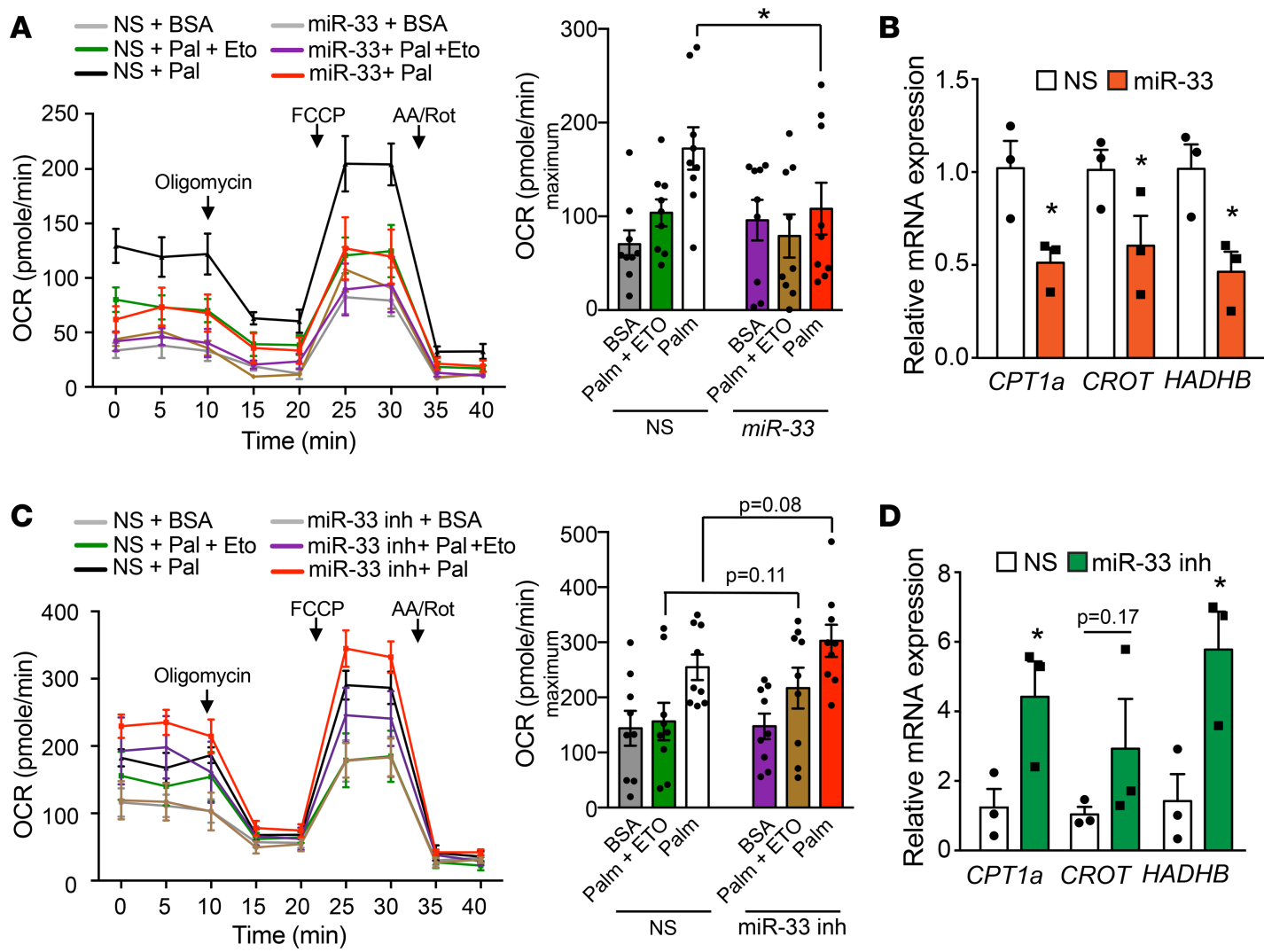

Figure 5. miR-33 regulates FAO in human kidney tubule cells. (A) Representative plots of Seahorse analysis of OCRs in the human tubular cell line HKC-8 transfected with CT or miR-33 mimics (left). Cells were treated with palmitate-BSA FAO substrate. Measurements were performed both under basal conditions and after treatment with the CPT1a inhibitor Eto to calculate the magnitude of several phases and parameters of mitochondrial respiration. Bar graphs show the OCRs associated with maximal respiratory status (right) ( $n=9$ replicate wells). (B) qRT-PCR analysis of the expression of miR-33 target genes involved in FAO, CPT1a, CROT, and HADHB, in HKC-8 cells transfected with CT or miR-33 mimics $(n=3)$. (C) Representative plots of Seahorse analysis of OCRs in HKC-8 cells transfected with CT or miR-33 inhibitors (left). Measurements were performed both under basal conditions and after treatment with Eto to calculate the magnitude of several phases and parameters of mitochondrial respiration. Bar graphs show the OCRs associated with maximal respiratory status (right) ( $n=9$ replicate wells). (D) qRT-PCR analysis of the expression of miR-33 target genes involved in FAO, CPT1a, CROT, and HADHB, in HKC-8 cells transfected with CT or miR-33 inhibitors $(n=3)$. Statistical significance was determined using 1-way ANOVA with Bonferroni's correction (A and $\mathbf{C}$ ) and nonparametric 2-tailed Mann-Whitney $U$ test (B and D). All data represent the mean $\pm S E M$ and ${ }^{*} P<0.05$ comparing HKC-8 cells transfected with miR-33 mimics/inhibitors with cells transfected with CT constructs under the same conditions.

following folic acid treatment, and this repression was abrogated in $m i R-33^{-1-}$ animals (Figure 4D). A similar protective effect on blunting the suppression of CPTla was observed in $m i R-33^{-1-}$ mice following UUO surgery (Figure 4E). To assess how miR-33 was mediating these effects, we first analyzed whether miR-33 is expressed in renal TECs and regulates lipid metabolism. Previous data from our laboratory (31) and miRNA tissue atlas database analysis (http://ccb-web.cs.uni-saarland.de/tissueatlas; Supplemental Figure 2A) show that miR-33 is expressed in mouse and human kidneys. Furthermore, analysis of the expression of miR-33 (miR-33a-5p in humans) levels in 400 human primary cells (fanton.grs.riken.jp; Supplemental Figure 2B) revealed that primary renal TECs isolated from 2 donors expressed high levels of miR-33 (only 9 and 13 human primary cell types had higher miR-33 expression out of 400 tested). We further supported the expression of miR-33 by qRT-PCR (Ct $=$ 23 ) in mouse primary renal TECs (Supplemental Figure 2C). Together these data indicate that miR-33 is present in renal TECs. To determine how miR-33 expression influences mitochondria bioenergetics, we isolated primary renal TECs from WT and $m i R-33^{-1-}$ mice and assessed the mitochondrial respiratory capacity. The results show that absence of miR-33 significantly enhanced the oxygen consumption rate (OCR). The effects of miR-33 deficiency on OCR were diminished in cells treated with the CPT1 inhibitor etomoxir, indicating that improved FAO is largely responsible for these effects (Figure $4 \mathrm{~F}$ ). Overall, these data suggest that the reduced fibrosis we observed in miR-33-deficient animals could be related to increased FAO and reduced lipid accumulation.

miR-33 regulates FAO in human renal TECs. We next sought to determine whether alterations in miR-33 expression could contribute to the development of kidney dysfunction in human cells. Using the HKC-8 
A

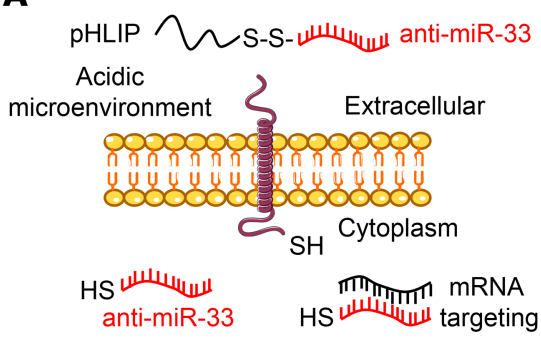

C
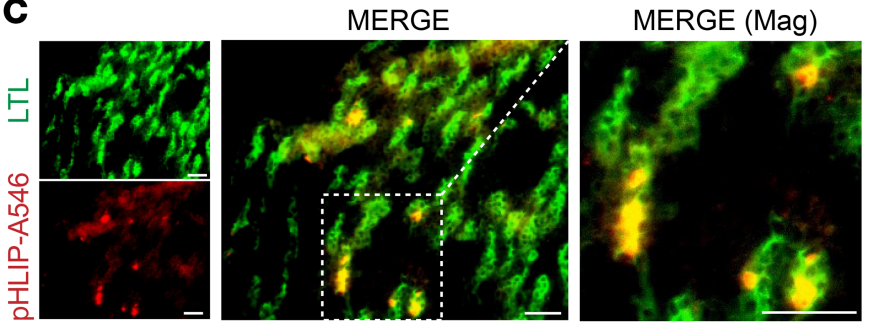

B

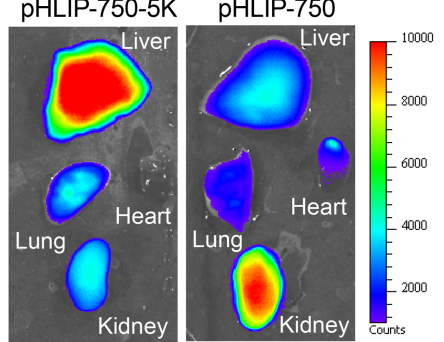

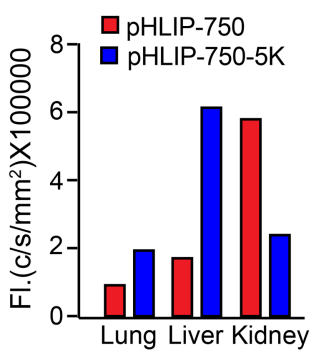

$\square$ Saline $\square$ pHLIP-A546

D

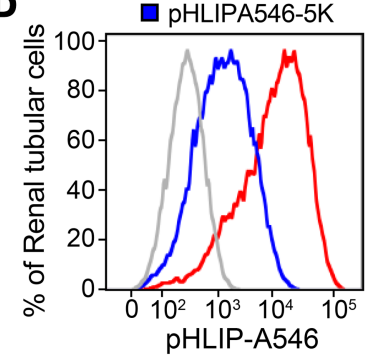

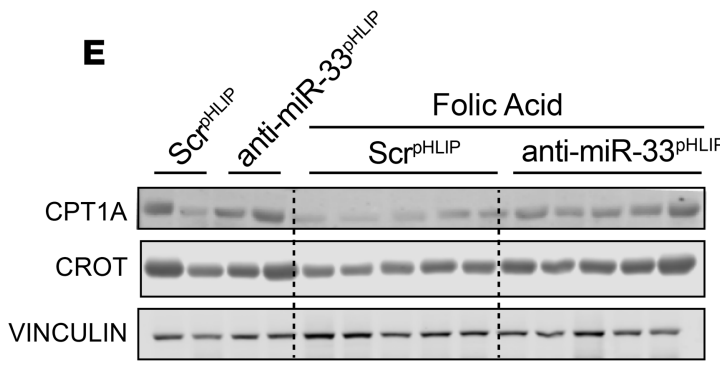
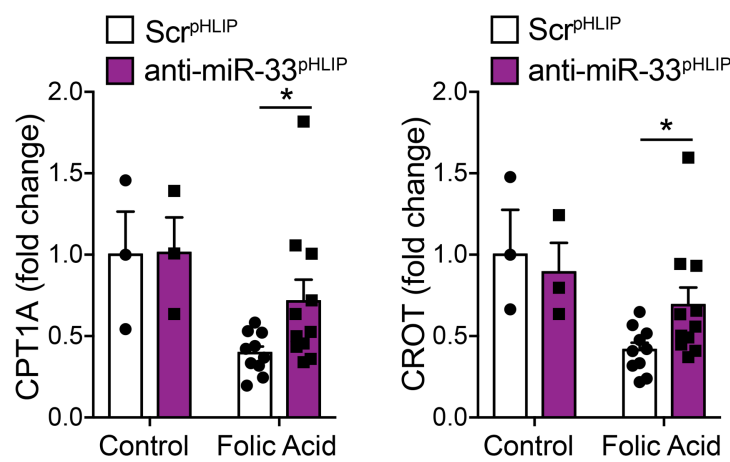

Figure 6. pH low inducible peptides promote delivery of miR-33 inhibitors to the kidney. (A) Generation of anti-miR-33 PNA-conjugated pH low insertion peptides (pHLIP) capable of delivering miR-33 inhibitors to acidic microenvironments. (B) Representative images of tissues from 1 mouse per group injected with fluorescently labeled noninserting CT (pHLIP-750-5K) or targeted (pHLIP-750) constructs. (C) Representative images demonstrating uptake of fluorescently labeled pHLIP constructs (pHLIP-A546) into primary renal TECs in vitro (shown in red). Costaining with lotus tetragonolobus lectin (LTL), a marker for proximal tubules is shown in green. (D) Representative flow cytometry histograms showing fluorescence intensities in primary renal tubular cells treated with CT (pHLIP-A546-5K), or targeted (pHLIP-A546) constructs. (E) Representative images and quantification of Western blot analysis of protein expression of miR-33 targets CPT1A and CROT in the kidneys of mice treated with pHLIP scrambled CT (ScrpHLIP) or miR-33 inhibitory RNA constructs (anti-miR-33 ${ }^{\text {HLLPP }}$ ) under CT conditions (top) or following treatment with FA ( $\left.n=10-11\right)$. Statistical significance was determined using nonparametric 2-tailed Mann-Whitney $U$ test. All data represent the mean \pm SEM and ${ }^{*} P<0.05$ comparing mice treated with anti-miR-33 ${ }^{\text {HLLP }}$ with animals treated with Scr ${ }^{\text {PHLIP }}$ under the same conditions. Scale bar: $20 \mu \mathrm{m}$.

human renal TEC line, we observed that the expression of miR-33 was not affected by treatment with the profibrotic cytokine TGF- $\beta$ (Supplemental Figure 3). We further demonstrate that treatment with miR-33 mimics is sufficient to reduce mitochondrial respiration in human kidney cells and that these effects are largely abolished by inhibition of FAO (etomoxir) (Figure 5A). Consistent with this, mRNA expression levels of CPT1a and other miR-33 targets involved in FAO (CROT, HADHB) were reduced in cells treated with miR-33 mimics (Figure 5B). Alternatively, treatment of HKC-8 cells with an inhibitor of miR-33 improved mitochondrial respiration (Figure 5C) and increased mRNA expression of miR-33 targets involved in FAO (Figure 5D).

Treatment with miR-33 inhibitors targeted to the kidney. We next assessed whether inhibition of miR-33 could be an effective therapeutic strategy to prevent folic acid-induced kidney damage. miR-33 inhibitors have previously been used as a strategy for raising circulating HDL-cholesterol and reducing atherosclerotic plaque size in animal models (32). However, long-term treatment with miR-33 inhibitors or genetic loss of miR-33 has been shown to result in detrimental unintended effects (33-35). As such, new strategies to deliver inhibitors to atherosclerotic plaques or disrupt binding of specific miR-33 targets could provide a more effective strategy. With this in mind, we have used the recently developed pHLIP, 
A

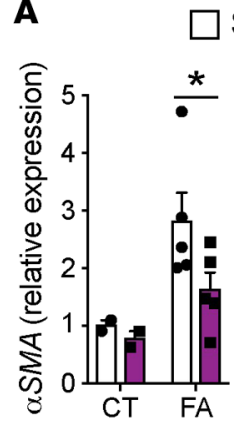

SCrPHLP

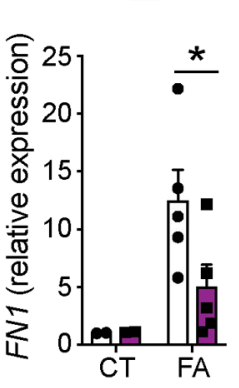

anti-miR-33pHLIP

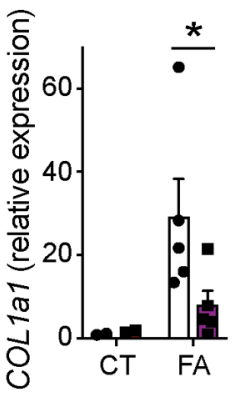

C

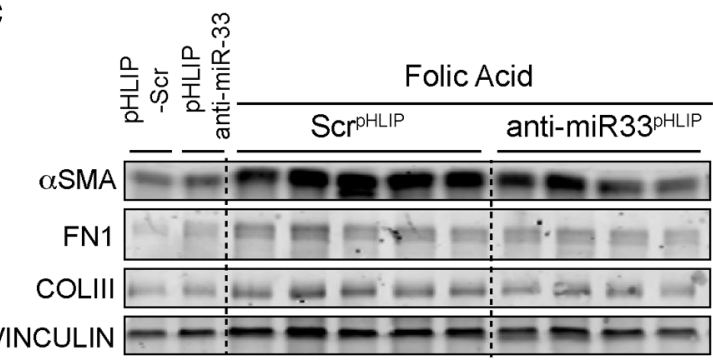

B

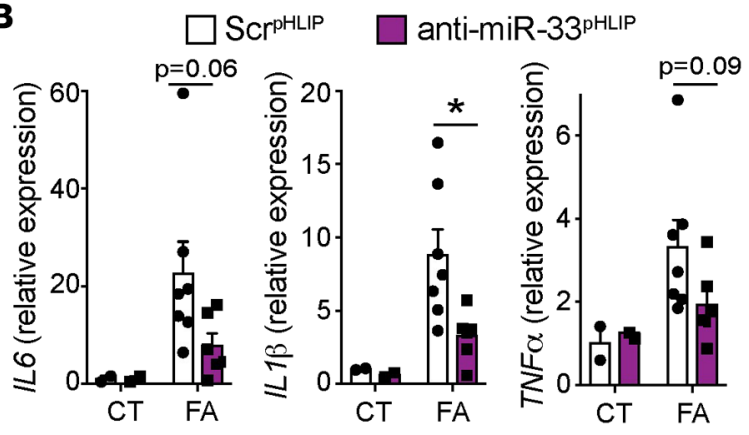

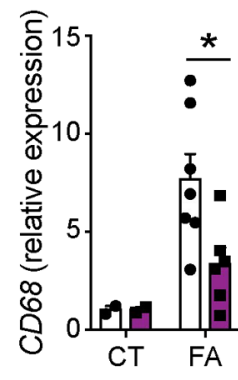
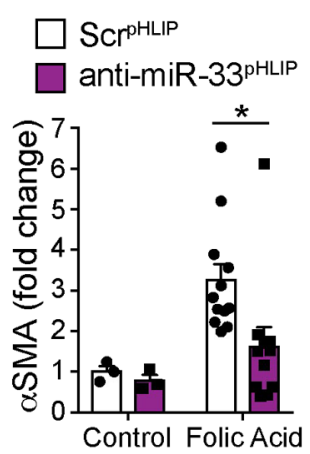

$\square$ ScrpHLIP
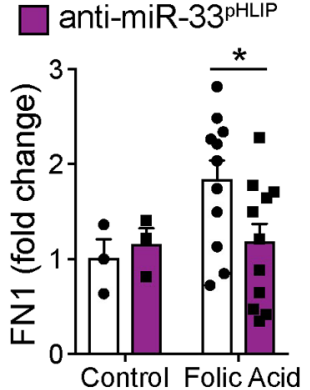

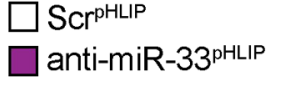

D

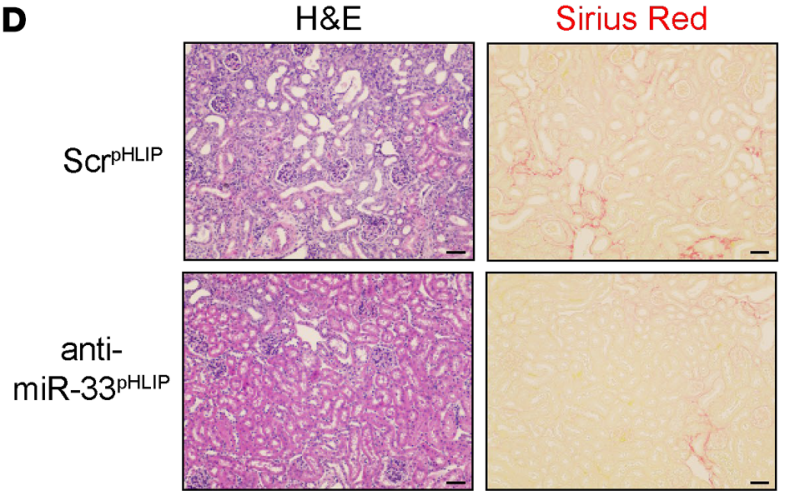

E

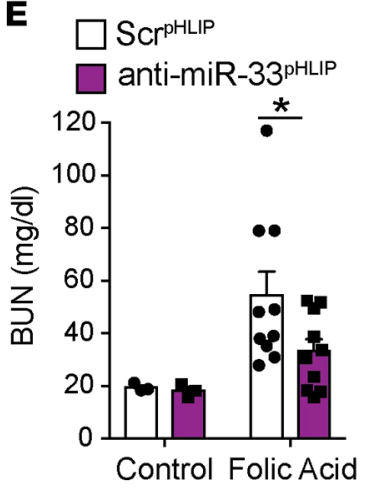

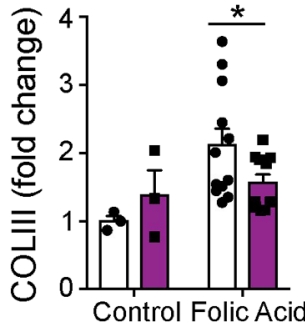

Figure 7. Selective delivery of miR-33 inhibitors abrogated FA-induced renal fibrosis. (A) qRT-PCR analysis of the expression of fibrotic genes ASMA, FN1,

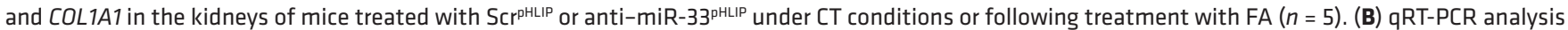

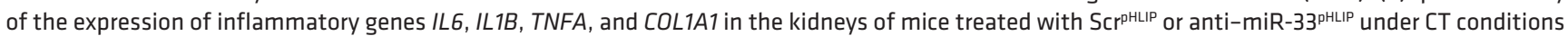
or following treatment with FA $(n=6-7)$. (C) Representative images and quantification of Western blot analysis of protein expression of fibrosis-associated genes: $\alpha$-SMA, FN1, and COLIII in the kidneys of mice treated with ScrpHLP or anti-miR-33 ${ }^{\text {pHLIP }}$ under CT conditions or following treatment with FA ( $n=$

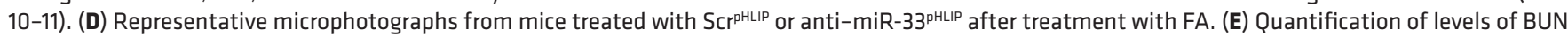

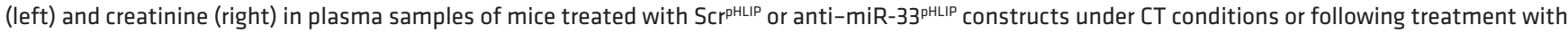
FA $(n=10-11)$. Statistical significance was determined using nonparametric 2-tailed Mann-Whitney $U$ test. All data represent the mean \pm SEM and ${ }^{*} P<$ 0.05 comparing mice treated with anti-miR-33 ${ }^{\text {pHLP }}$ with animals treated with Scr ${ }^{\text {PHLIP }}$ under the same conditions. Scale bar: $100 \mu \mathrm{m}$.

which can deliver miRNA inhibitors, or other small oligonucleotides, selectively to areas of high acidity. This strategy has previously been employed to deliver miRNA inhibitors to the acidic microenvironment of tumors (36). The capacity of some portions of the mammalian nephron to secrete hydrogen ions (37) theoretically provides an acidic microenvironment. This, together with the fact that original reports have documented pHLIP renal accumulation (38), led us to develop anti-miR-33 ${ }^{\text {pHLIP }}$ PNA constructs to inhibit miR-33 in the kidney (Figure 6A). Assessment of fluorescently labeled constructs (TAMRA dye) confirmed that whereas standard delivery systems and noninserting control constructs (pHLIP-750-K) convey inhibitors primarily to the liver, functional pHLIP constructs (pHLIP-750) have extremely high uptake in the kidney (Figure 6B). Furthermore, we demonstrate uptake of fluorescent pHLIP constructs in primary renal tubular cells by both fluorescent microscopy (Figure 6C) and flow cytometry (Figure 6D and Supplemental Figure 4). Notably, we demonstrate that treatment with antimiR-33 $3^{\text {pHLIP }}$ constructs significantly increases protein levels of CPT1a as well as CROT, another miR-33 
target involved in FAO (Figure 6E). However, the expression of other known targets of miR-33 that are involved in FAO and mitochondrial function, including PGC-1 $\alpha$ and AMPK, were not found to be altered in the kidneys of these mice (Supplemental Figure 5).

Importantly, we found that treatment with anti-miR-33 ${ }^{\text {pHLIP }}$ PNA constructs can substantially reduce folic acid-induced kidney fibrosis. Similar to what we observed in $m i R-33^{-1-}$ animals, treatment with antimiR-33 ${ }^{\text {pHLIP }}$ constructs reduced the induction of genes involved in both fibrosis (Figure 7A) and inflammation (Figure 7B) in the kidney following folic acid treatment compared with scramble control PNAs (Scr ${ }^{\text {HLIP }}$ ). Similarly, the expression of proteins involved in fibrosis was significantly reduced in animals treated with anti-miR-33 ${ }^{\text {HLIP }}$ constructs (Figure 7C). We also performed histological staining of kidney sections from these animals (Figure 7D) and assessed circulating factors associated with renal function, demonstrating a significant decrease in circulating BUN and a similar trend for creatinine (Figure 7E).

\section{Discussion}

Although there are many causes of kidney dysfunction, alterations in metabolic function play an important role in both the development and progression of $\mathrm{CKD}$, including DN and tubulo-interstitial fibrosis, the latter being the final common pathway for hypertensive renal disease and nephrosclerosis. Indeed, one of the most common conditions in patients with diabetes mellitus is DN, which in turn is one of the largest contributors to ESRD. In addition to ESRD, patients with DN are also much more likely to suffer from events associated with cardiovascular disease (CVD) (39). Although very distinct, CKD and CVD are both chronic inflammatory conditions associated with altered lipid metabolism (40). miR-33 is a well-known mediator of lipid metabolism and a promising target for the treatment of CVD (41). In this work, we show that loss of miR-33 protects against the development of kidney fibrosis, demonstrating the potential for anti-miR-33 therapeutics that can target both CKD and CVD in patients suffering ESRD.

By using 2 models of renal injury (folic acid and UUO), we show that the genetic ablation of miR-33 protects from the development of renal fibrosis. In addition, the presence of proinflammatory markers typical of early fibrogenesis was also reduced. Inflammation contributes significantly to fibrogenesis (42), and miR-33 has been reported to influence macrophage polarization and shown to play an important role in the development of other chronic inflammatory conditions, such as atherosclerosis. Additional evidence suggests that miR-33 facilitates renal fibrosis in DN through the activation of the NF- $\kappa$ B/TGF- $\beta$ inflammation pathway (28). However, our data from BMT experiments demonstrate that the protection from kidney fibrosis observed in $m i R-33^{-1-}$ mice was not due to differences in circulating leukocytes, but rather to the local effect of miR-33 on TECs and other cells within the kidney. Because our work focuses on the metabolic facet of renal fibrosis and the action of miR-33 in TECs, we have not explored in depth the role of resident macrophages, which are important contributors to renal fibrogenesis and would unlikely be replaced following BMT $(43,44)$. Also, this work has not addressed whether miR-33 impinges on other pathogenic pathways related to CKD and fibrogenesis, such as epithelial-mesenchymal transition $(45,46)$.

Excessive lipid accumulation and impaired FAO have been repeatedly observed in several animal models of acute and chronic renal injury, as well as in human patients $(10,11,13,47,48)$. Increased lipid accumulation induces cellular lipotoxicity, contributing to fibrosis development (11). Although cell-specific overexpression of CD36 in TECs from mice leads to lipid accumulation, it is not sufficient to drive spontaneous renal fibrogenesis (49). Therefore, it appears that defects in energy production have detrimental consequences beyond mere lipid accumulation. miR-33 may also promote the accumulation of lipid droplets in the kidney as described in other organs, thus contributing to kidney damage (33). The importance of impaired mitochondrial respiration as a forerunner of tubular damage is also underscored by the fact that mitochondrial substrate overload surpasses the catabolic capacity of mitochondrial $\beta$-oxidation and promotes tubular injury $(12,50)$. A decrease in the expression of key metabolic transcription factors (PPARGC1A, PPARA, and $P P A R G$ ) in tubule cells (15) has been associated with the progression of fibrosis and CKD. Transgenic expression of PPARGC1A and PPARA caused protection from acute kidney injury or kidney fibrosis, indicating the key role of metabolic pathways in maintaining kidney function and tubule health $(10,13,51,52)$. Furthermore, age-associated impairment of PPAR $\alpha$ and FAO aggravates renal fibrosis (53). Consistent with this, the pharmacological activation of PPAR $\alpha$ by its agonist fenofibrate not only improved FAO but also significantly reduced renal fibrosis development in the UUO and folic acid models. In addition, treatment with C75, a synthetic compound that increases CPT1 activity and blocks fatty acid synthase, attenuated kidney fibrosis 
associated with FA administration (11). This molecule was also protective in the ischemia/reperfusion injury model, where stimulation of CPT1 activity by C75 recovered ATP depletion, improved renal function, attenuated tissue injury, and inhibited proinflammatory cytokine production and neutrophil infiltration (54). Nevertheless, neither fibrates nor C75 are currently accepted therapies for CKD either because of side effects on renal function or lack of pharmaceutical development. As such, novel treatments aimed at preventing CKD-associated metabolic alterations could provide a promising approach for the treatment of CKD.

miR-33 has been demonstrated to be a key regulator of FAO because of its ability to regulate key enzymes in this process, including CPT1a. TECs rely exclusively on FAO and are unable to switch to glycolysis in response to energy demand. Thus, pharmacological inhibition of CPT1 results in ATP depletion, cell death, and dedifferentiation (11). In line with these observations, we observed that FAO-associated mitochondrial respiration is increased in renal TECs from $m i R-33^{-1-}$ animals. These effects were greatly diminished in cells treated with etomoxir, indicating that these changes are largely due to the action of miR-33 on FAO. Other miR-33 targets involved in regulating mitochondrial function and glucose metabolism could also contribute to the improved respiratory capacity of TECs from $m i R-33^{-/-}$animals and the trend toward increased respiration even after inhibition of CPT1a. The improved FAO in these cells was reflected in an increased capacity of kidney tissue from $m i R-33^{-1-}$ mice to oxidize ${ }^{14} \mathrm{C}$-palmitate. Consistent with this, the expression of CPT1a was increased in these mice following renal injury, and the accumulation of lipids in the kidneys of these animals was dramatically reduced. Furthermore, our results in human kidney cells are also strongly supportive of a potential role for miR-33 in human kidney fibrosis. Studies of mitochondrial respiration and ATP production in these cells demonstrated similar effects on FAO, exemplified by the changes in the expression of CPT1a, as well as other miR-33 targets involved in FAO (CROT and $H A D H B)$. These findings suggest that treatment of human patients with inhibitors of miR-33 could increase the expression of genes involved in FAO and reduce lipid accumulation, which could help protect against kidney dysfunction.

miRNAs have emerged as powerful regulators of fibrotic processes in the kidney $(55,56)$. Thus, Gomez et al. identified 24 miRNAs that were commonly upregulated both in human CKD and in animal models of kidney injury, suggesting a "fibrotic" miRNA signature in the kidney (18). miR-21 is arguably the first miRNA shown to promote fibrosis by targeting PPAR $\alpha$-related metabolic pathways (22). Anti-miRNA-21 oligonucleotide-based therapy has also confirmed this finding in the murine model of Alport nephropathy by reversing the associated mitochondrial dysfunction (17). Although the mechanism of action of miR214 in fibrosis is less well elucidated, it appears that miR-214 also targets metabolic responses (18). Earlier work has suggested that miR-33 may play a role in the regulation of hepatic fibrosis, as it was reported that inhibition of miR-33 reduces the mRNA expression of some fibrosis-associated factors in a nonalcoholic steatohepatitis model (57). A profibrotic role for miR-33 has been also reported in cardiac fibrosis, as cardiac fibroblast-specific deficiency of miR-33 reduced cardiac fibrosis in response to pressure overload by transverse aortic constriction. The proposed mechanism underlying this response was that deficiency of miR-33 impaired cardiac fibroblast proliferation because of altered lipid raft cholesterol content $(58,59)$. In the kidney, we demonstrate an important role for miR-33 in the regulation of FAO in TECs, which may be relevant to explain its effects on fibrogenesis. However, it is likely that other known or predicted miR-33 targets may also be involved in helping promote these actions.

Because of their important role in the regulation of metabolic function and other pathways related to fibrosis, miR-33 and other miRNAs could provide useful therapeutic targets for kidney disease. However, the promiscuous nature of miRNAs increases the potential for unintended consequences, as has previously been observed with long-term treatment with inhibitors of miR-33 (33). Therefore, the ability to target miRNA mimics and inhibitors to specific tissues could greatly enhance their therapeutic potential. In this work, we used pHLIP technology to selectively deliver miR-33 inhibitors to the kidney, based on their affinity for acidic microenvironments (36). We observed efficient uptake into the kidney and demonstrated that selective delivery of miR-33 inhibitors to the kidney is sufficient to reduce folic acid-induced kidney damage. These findings highlight the therapeutic potential of anti-miR-33 ${ }^{\text {pHLIP }}$ constructs for the treatment of $\mathrm{CKD}$, while minimizing the potential risks of detrimental effects in other organs. Conceivably, a similar approach can be used to selectively target other profibrotic miRNAs or deliver protective miRNAs into the kidney. Hence, these findings highlight the potential for the selective targeting of kidney miRNAs as a therapy for patients with CKD. 


\section{Methods}

Animals, BM transplantation, and models of renal fibrosis

Male C57BL/6 (WT) mice were originally purchased from The Jackson Laboratory (Bar Harbor, Maine, USA) and kept under constant temperature and humidity in a controlled 12-hour light/12-hour dark cycle. $m i R-33^{-/-}$mice were generated and characterized, and BMTs were performed as we have previously described (35). Briefly, 8-week-old male mice were lethally irradiated twice 4 hours apart with a total dose of 5.5 Gy (550 rads) before transplantation (X-RAD320, Precision X-Ray Inc.). BM was collected from

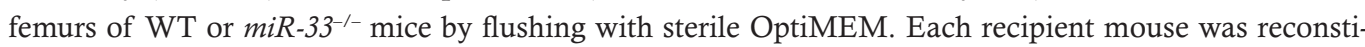
tuted with $2 \times 10^{6} \mathrm{BM}$ cells through retroorbital injection. Four weeks after BMT, peripheral blood was collected by retroorbital venous plexus puncture for PCR analysis of BM reconstitution.

\section{Mouse models of kidney fibrosis}

UUO. UUO surgery procedure was performed as previously described (60). Briefly, mice were anesthetized with isoflurane ( $3 \%-5 \%$ for induction and $1 \%-3 \%$ for maintenance). Mice were shaved on the left side of the abdomen, a vertical incision was made through the skin with a scalpel, and the skin was retracted. A second incision was made through the peritoneum to expose the kidney. The left ureter was ligated twice $15 \mathrm{~mm}$ below the renal pelvis with surgical silk, and the ureter was then severed between the 2 ligatures. Then, the ligated kidney was placed gently back into its correct anatomical position, and sterile saline was added to replenish loss of fluid. The incisions were sutured and mice were individually caged. Buprenorphine was used as an analgesic. A first dose was administered 30 minutes before surgery and then every 12 hours for 72 hours, at a dose of $0.05 \mathrm{mg} / \mathrm{kg}$ subcutaneously. Mice were sacrificed by $\mathrm{CO}_{2}$ overdose, and kidney and blood samples were harvested after perfusion with PBS at 3 and 10 days after UUO. Contralateral kidneys were used as a nonfibrotic control for all experiments using this model.

$F A N$. In this model kidney fibrosis was induced by i.p. injection with $250 \mathrm{mg}$ folic acid per kilogram body weight dissolved in $0.3 \mathrm{M}$ sodium bicarbonate (vehicle) as previously described (61). Control animals received $0.3 \mathrm{~mL}$ of vehicle (i.p.). Mice were sacrificed by $\mathrm{CO}_{2}$ overdose, and kidneys and blood samples were harvested after perfusion with PBS after 7 days of FA administration.

\section{Histology, immunohistochemistry, and morphometric analyses}

Kidneys were fixed in $10 \%$ neutral buffered formalin overnight, bisected sagittally, and submitted for standard paraffin processing before sectioning at $5 \mu \mathrm{m}$. Sections were stained with H\&E, or Sirius red, to evaluate extent of renal injury and associated fibrosis. To quantify fibrosis, 20 randomly selected fields across 4 sections, evenly distributed from pole to pole, limited to the cortex and avoiding preexisting perivascular and capsular collagen, were captured with a $\times 20$ objective using a polarizing filter and Leica DFC 495 digital camera connected to a light microscope (Zeiss Axioskop). The area occupied by collagen was measured in each field using a computerized image analysis system (Leica Application Suite X, Leica Microsystems Inc.) and expressed in square micrometers. The pathologist was blinded to genotype and treatment status of individual animals.

\section{Cell culture}

Human proximal TECs (HKC-8) were cultured in DMEM/F12 (1:1 vol/vol) supplemented with $15 \mathrm{mM}$ HEPES, $5 \%$ (vol/vol) fetal bovine serum, $1 \times$ insulin-transferrin-selenium, $0.5 \mu \mathrm{g} / \mathrm{mL}$ hydrocortisone, 50 $\mathrm{U} / \mathrm{mL}$ penicillin, and $50 \mu \mathrm{g} / \mathrm{mL}$ streptomycin at $37^{\circ} \mathrm{C}$ and $5 \% \mathrm{CO}_{2}$. HKC-8 cells were provided by Katalin Susztak (Department of Medicine, Perelman School of Medicine, University of Pennsylvania, Philadelphia, Pennsylvania, USA). HKC-8 cells were transfected with $40 \mathrm{nM}$ miR-33 mimic or $60 \mathrm{nM}$ miR-33 inhibitor using RNAiMAX (Thermo Fisher Scientific) for 8 hours, as described previously (62). All experimental control samples were treated with an equal concentration of a nontargeting control mimic sequence or an inhibitor negative control sequence. Between 48 and 72 hours after transfection, cells were collected for bioenergetic or mRNA analysis.

\section{Isolation of primary kidney TECs}

Kidneys from $m i R-33^{--}$and WT mice were collected after sacrifice and subjected to PBS perfusion (10 $\mathrm{mL}$ ) followed by collagenase type II digestion $(2 \mathrm{mg} / \mathrm{mL}, 10 \mathrm{~mL})$. After digestion, the renal capsule and medulla was removed. The rest of the kidney was minced and further digested in collagenase buffer for 
an additional 5 minutes at $37^{\circ} \mathrm{C}$ with rotation to release cells. Digested tissue and cell suspension were passed through a $70-\mu \mathrm{m}$ cell strainer, centrifuged at $50 \mathrm{~g}$ for 5 minutes, and washed in PBS for 2 rounds to collect TECs. Isolated TECs were seeded onto a collagen-coated Petri dish and cultured in renal epithelial cell medium (C-26130, PromoCell) supplemented with growth factors, including insulin, epinephrine, and epidermal growth factor for TEC growth, as well as hydrocortisone for limiting growth of potential contaminating macrophages derived from the isolation (63).

\section{Cellular bioenergetic analysis}

FAO-associated OCR was studied using high-throughput extracellular flux analysis (Seahorse XFe96 and XFe24 analyzers, Agilent Technologies). Ex vivo, primary TECs isolated from WT or $m i R-33^{-1-}$ mice were seeded onto collagen-coated XFe96 cell culture microplates 24 hours before extracellular flux analysis. Growth medium was replaced with substrate-limited medium (DMEM supplemented with $0.5 \mathrm{mM}$ glucose and $1 \mathrm{mM}$ glutamate) the night before Seahorse assay. On the assay day, substrate-limited medium was replaced with Krebs-Henseleit buffer assay medium supplemented with $0.2 \%$ carnitine for 1 hour at $37^{\circ} \mathrm{C}$ without $\mathrm{CO}_{2}$. The CPT1 inhibitor etomoxir (MilliporeSigma) was added $(4 \mu \mathrm{M})$ to the corresponding wells 15 minutes before starting the assay. Finally, just before starting the assay, BSA or $200 \mathrm{mM}$ palmitate-BSA FAO substrate was added.

Immediately, XF Cell Mito Stress Test was performed in a Seahorse XFe96 analyzer. FAO was assayed through measurement of OCR changes in response to sequential injections of 4 compounds: oligomycin (1 $\mu \mathrm{M}$, port A), FCCP (1 $\mu \mathrm{M}$, port B), and antimycin/rotenone (1 $\mu \mathrm{M}$, port C). After Seahorse assay, Hoechst staining was performed for data normalization.

In vitro, $2 \times 10^{4} \mathrm{HKC}-8$ cells were transfected with miR-33 mimics, miR-33 inhibitors, or corresponding negative control sequences. Cells were then seeded in a Seahorse Bioscience XFe24 cell culture microplate, followed by the abovementioned preparation steps, and given $400 \mu \mathrm{M}$ etomoxir treatment before OCR measurements in response to oligomycin ( $1 \mu \mathrm{M}$, port $\mathrm{A})$, FCCP $(3 \mu \mathrm{M}$, port $\mathrm{B})$, and antimycin/ rotenone $(1 \mu \mathrm{M}$, port $\mathrm{C})$ in an XFe24 analyzer. After the assay, protein was extracted from wells with $0.1 \%$ NP-40-PBS solution and quantified with bicinchoninic acid protein assay (Thermo Fisher Scientific) for data normalization. OCR and maximal respiration were determined as described previously $(63,64)$.

\section{Kidney TAG measurement}

Renal tissue lipids were sequentially extracted by the method of Bligh and Dyer (65) in the presence of internal standards, including di-eicosanoyl-PC, di-tetradecanoyl-PE, and cholesteryl heptadecanoate. Extracted renal lipids were resuspended in methanol/chloroform (4:1, by vol) to a dilution of about 20 pmol of lipid per microliter and analyzed by electrospray ionization mass spectrometry in the direct infusion mode at a flow rate of $3 \mu \mathrm{L} /$ min using a Thermo Fisher Scientific Electron TSQ Quantum Ultra instrument.

\section{FAO}

FAO was assayed as previously described (66). In brief, kidneys were removed from WT and $m i R-33^{-1-}$ mice and homogenized in 5 volumes of chilled STE buffer ( $\mathrm{pH} 7.4,0.25 \mathrm{M}$ sucrose, $10 \mathrm{mM}$ Tris-HCl, and 1 mM EDTA). The homogenate was immediately centrifuged and the pellet was resuspended and incubated with a reaction mixture containing $0.5 \mathrm{mmol} / \mathrm{L}$ palmitate (conjugated to $7 \% \mathrm{BSA} /\left[{ }^{14} \mathrm{C}\right]$ palmitate at 0.4 $\mu \mathrm{Ci} / \mathrm{mL}$ ) for 30 minutes. After this incubation period, the resuspended pellet-containing reaction mixture was transferred to an Eppendorf tube, the cap of which housed a Whatman filter paper disc that had been presoaked with $1 \mathrm{~mol} / \mathrm{L}$ sodium hydroxide. The ${ }^{14} \mathrm{CO}_{2}$ trapped in the reaction mixture medium was then released by acidification of medium using $1 \mathrm{~mol} / \mathrm{L}$ perchloric acid and gentle agitation of the tubes at $37^{\circ} \mathrm{C}$ for 1 hour. Radioactivity that had become adsorbed onto the filter disc was then quantified by liquid scintillation counting in a beta counter.

\section{Creatinine and BUN measurements}

Briefly, creatinine was extracted from $20 \mu \mathrm{L}$ of serum via a protein precipitation crash out using acetonitrile. The resulting extraction was then spiked with internal deuterated creatinine and analyzed via Ultra High Pressure Liquid Chromatography System (Agilent Technologies) coupled in-line to a 4000 Q-Trap mass spectrometer (AB Sciex). The extract was injected onto an Agilent Technologies column coupled to an analytical Phenomenex SecurityGuard trap $(\mathrm{C} 18,4 \times 3.0 \mathrm{~mm})$. The column and trap were kept at $40^{\circ} \mathrm{C}$. 
Bound creatinine was eluted at a flow rate of $500 \mu \mathrm{L} /$ min using an acetonitrile/water mobile phase mixture containing $0.1 \%$ formic acid. First quartile to $(\mathrm{m} / z 114)$ third quartile $(\mathrm{m} / z 44)$ and first quartile $(\mathrm{m} / z 117)$ to third quartile $(\mathrm{m} / z 47)$ transitions were monitored for $\mathrm{H}_{3}$-creatinine (serum sample) and $\mathrm{D}_{3}$-creatinine (reference internal standard) abundance, respectively. Mass spectral data were collected and analyzed/quantified using Analyst software (v.1.5). Standard curves of $\mathrm{D}_{3}$-creatinine were used to ensure linearity within the creatinine measurements in the serum samples. Quantitative colorimetric determination of urea nitrogen in plasma was performed using the diacetylmonoxime (DAM) method and measured at $520 \mathrm{~nm}$ on the Excel Chemistry Analyzer (Stanbio Laboratory, Boerne, Texas, USA).

\section{Western blot analysis}

Kidneys were homogenized by manual disruption and the Bullet Blender Homogenizer and lysed in icecold buffer containing $50 \mathrm{mM}$ Tris- $\mathrm{HCl}$ at $\mathrm{pH} 7.5,0.1 \%$ SDS, $0.1 \%$ deoxycholic acid, $0.1 \mathrm{mM}$ EDTA, $0.1 \mathrm{mM}$ EGTA, 1\% NP-40, $5.3 \mathrm{mM} \mathrm{NaF}, 1.5 \mathrm{mM} \mathrm{NaP}, 1 \mathrm{mM}$ orthovanadate, and $1 \mathrm{mg} / \mathrm{mL}$ protease inhibitor cocktail (Roche) and $0.25 \mathrm{mg} / \mathrm{mL}$ AEBSF (Roche). Lysates were sonicated and rotated at $4^{\circ} \mathrm{C}$ for 1 hour before the insoluble material was removed by centrifugation at 12,000 $\mathrm{g}$ for 10 minutes. After normalizing for equal protein concentration, cell lysates were resuspended in SDS sample buffer before separation by SDS-PAGE. Following transfer of the proteins onto nitrocellulose membranes, the membranes were probed with the following antibodies: ABCA1 (Abcam; ab18180; 1:1000), CROT (Novus; 3144; 1:1000), CPT1a (Abnova; H00001374-DO1P; 1:1000), GAPDH (AF5718; 1:2000), $\alpha$-SMA (MilliporeSigma; A5228; 1:2000), FN1 (MilliporeSigma; F3648; 1:1000), COLIII (Abcam; ab7778; 1:1000), and VINCULIN (MilliporeSigma; V9131; 1:2000). Protein bands were visualized using the Odyssey Infrared Imaging System (LI-COR Biotechnology), and densitometry was performed using ImageJ software (NIH).

\section{RNA isolation and qRT-PCR}

Total RNA from cells and tissue was isolated using TRIzol reagent (Invitrogen, Thermo Fisher Scientific) according to the manufacturer's protocol. For mRNA expression analysis, cDNA was synthesized using iScript RT Supermix (Bio-Rad), following the manufacturer's protocol. qRT-PCR analysis was performed in duplicate using SsoFast EvaGreen Supermix (Bio-Rad) on an iCycler Real-Time Detection System (Eppendorf). The mRNA levels were normalized to 18S. Quantitative PCR primers are listed in Supplemental Table 1.

\section{PNA synthesis and pHLIP-PNA conjugate synthesis}

pHLIP-PNA conjugations were performed as previously described (38). First, pHLIP-Cys (ADDQNPWRAYLDLLFPTDTLLLDLLWCG, CS Bio) was conjugated with Aldrithiol-2 (MilliporeSigma) to get pHLIP-Cys(Pys). Aldrithiol-2 was added to peptide in dimethylformamide (DMF) to have a molar ratio of 1:1. One hundred millimoles sodium phosphate and $150 \mathrm{mM} \mathrm{NaCl}$ buffer, $\mathrm{pH} 7.2$ (saturated with argon), was added to the reaction mix (one-twentieth of total volume). Reaction mixture was incubated at room temperature for 1 hour. pHLIP-Cys(Pys) was purified by reverse-phase HPLC (Zorbax SB-C18 columns, $9.4 \times 250 \mathrm{~mm}, 5$ $\mu \mathrm{m}$; Agilent Technologies, the gradient consisting of a binary solvent system using water and acetonitrile with $0.05 \%$ trifluoroacetic acid [TFA] for $20 \%-70 \%$ over 40 minutes), lyophilized, and characterized by surface-enhanced laser desorption/ionization time-of-flight (SELDI-TOF) mass spectrometry. Second, PNAs were added to pHLIP-Cys(Pys) in DMSO (molar ratio 1:3) in the presence of $0.1 \mathrm{M}$ ammonium bicarbonate, $\mathrm{pH} 8.8$ (saturated with argon), one-fifth of total volume, and the reaction mixture was incubated overnight at $30^{\circ} \mathrm{C}$. $\mathrm{pHLIP}$ PNA was purified by reverse-phase HPLC (Zorbax SB-C18 columns, $9.4 \times 250 \mathrm{~mm}, 5 \mu \mathrm{m}$; Agilent Technologies, the gradient consisting of a binary solvent system using water and acetonitrile with $0.05 \%$ TFA for $5 \%-65 \%$ over 40 minutes), lyophilized, and characterized by SELDI-TOF mass spectrometry.

pHLIP and K-pHLIP conjugation with Alexa Fluor 750 and Alexa Fluor 546 fluorescent dye pHLIP (ACDDQNPWRAYLDLLFPTDTLLLDLLWA) and K-pHLIP (ACKKQNPWRAYLKLLFPTKTLLL KLLW) were conjugated at the N-terminus with Alexa Fluor 546- and Alexa Fluor 750-maleimide (Life Technologies) in DMF at a ratio of 1:1 at room temperature for about 2 hours. One hundred millimoles sodium phosphate and $150 \mathrm{mM} \mathrm{NaCl}$ buffer, $\mathrm{pH} 7.2$ (saturated with argon), was added to the reaction mix (one-twentieth of total volume). The reaction progress was monitored by the analytical reverse-phase HPLC (Zorbax SB-C18 column, $4.6 \times 250 \mathrm{~mm}, 5 \mu \mathrm{m}$; Agilent Technologies; the gradient consisting of a binary 
solvent system using water and acetonitrile with $0.05 \%$ TFA for $20 \%-75 \%$ over 30 minutes). More Alexa Fluor-maleimide was added to the reaction mix, if it was needed. Constructs were purified by reverse-phase HPLC (Zorbax SB-C18 columns, $9.4 \times 250 \mathrm{~mm}, 5 \mu \mathrm{m}$; Agilent Technologies, the same gradient, over 40 minutes), lyophilized, and characterized by SELDI-TOF mass spectrometry. The concentrations of the constructs were determined by their absorbance using the following molar extinction coefficients: $\varepsilon_{554}=93,000$ $\mathrm{M} / \mathrm{cm}$ (for Alexa Fluor 546-pHLIPs), and $\varepsilon_{753}=290,000 \mathrm{M} / \mathrm{cm}$ (for Alexa Fluor 750-pHLIPs).

\section{Assessment of pHLIP delivery}

To determine the specificity of pHLIP delivery, animals were injected intravenously with pHLIP-750 or the noninserting control peptide pHLIP-750-5K ( $4 \mathrm{nmol})$. Animals were sacrificed after 12 hours and uptake was determined in different tissues by near-infrared fluorescence imaging, performed on an IVIS Spectrum system (Caliper Life Science) with appropriate excitation (Ex) and emission (Em) filter sets $(\mathrm{Ex} / \mathrm{Em}=$ $745 / 800 \mathrm{~nm}$ ). Additionally, kidney sections were blocked with 10\% donkey serum for 30 minutes and stained with LTL (FL1321, Vector Laboratories) for 30 minutes at room temperature for localization of renal epithelial cells (67). For flow cytometry analysis, kidneys were harvested 4 hours after injection with pHLIP-546-5K or pHLIP-546, and cell dissociation was performed as described for isolation of kidney epithelial cells. A mixture of kidney cells was passed through a $70-\mu \mathrm{m}$ cell strainer, and kidney epithelial cells were stained with LTL (FL1321, Vector Laboratories). Flow cytometry was performed using a BD LSRII (BD Biosciences), and data were analyzed using FlowJo software v8.7 (Tree Star, Inc.).

Anti-miR-33 ${ }^{\text {pHLIP }}$ treatment

$\mathrm{Scr}^{\mathrm{pHLIP}}$ and anti-miR-33 ${ }^{\mathrm{pHLIP}}$ constructs were administered by intravenous injection at a dose of $1 \mathrm{mg} / \mathrm{kg}$ body weight in PBS with 5\% DMSO. Injections were performed 2 days before, the day of, 2 days after, and 5 days after administration of folic acid.

\section{Statistics}

Animal sample size for each study was chosen based on literature documentation of similar well-characterized experiments. The number of animals used in each study is listed in the figure legends. In vitro experiments were routinely repeated at least 3 times unless otherwise noted. No inclusion or exclusion criteria were used. Data are expressed as average \pm SEM. Statistical differences were measured using an unpaired 2 -sided Student's $t$ test or 1-way ANOVA with Bonferroni's correction for multiple comparisons. Normality was checked using the Kolmogorov-Smirnov test. A nonparametric test (Mann-Whitney) was used when data did not pass the normality test. $P \leq 0.05$ was considered statistically significant. Data analysis was performed using GraphPad Prism software version 7.

\section{Study approval}

All the experiments were approved by the Institutional Animal Care Use Committee of Yale University School of Medicine.

\section{Author contributions}

NLP, VM, CFH, and SL conceived and designed the study and wrote the manuscript. NLP, SL, VM, WD, AKS, NR, AM, OAA, JT, CZ, MMS, NA, and AB performed experiments and analyzed data. SM, YKR, and RB synthesized and characterized the anti-miR-33 PNAs. YS and DRP assisted with experimental design and data interpretation.

\section{Acknowledgments}

We thank Gordon Terwilliger and Michael Schadt for the technical support in preparation of renal tissues through the Anatomic Pathology Phenotyping Core in the Department of Comparative Medicine, Yale University School of Medicine. This work was supported by grants from the NIH (R35HL135820 to CFH, R01HL105945 and R01HL135012 to YS, GM073857 to OAA and YKR, and P30 DK079310 to the George M. O'Brien Kidney Center at Yale), the American Heart Association (16EIA27550005 to CFH, 16GRNT26420047 to YS, and 17SDG33110002 to NR), the Foundation Leducq Transatlantic Network of Excellence in Cardiovascular Research MicroRNA-based Therapeutic Strategies in Vascular Disease (to CFH), and the University of Connecticut START PPOC award (to RB). This work was also supported by grants 
from the Ministerio de Economía y Competitividad (MINECO) (SAF2015-66107-R to SL), Carlos III Health Institute (ISCIII) European Regional Development Fund (PI17/01513 to DRP); cofunded by the European Regional Development Fund; and supported by Instituto de Salud Carlos III REDinREN RD12/0021/0009 and RD16/0009/0016 (to SL and DRP), Comunidad de Madrid "NOVELREN" B2017/BMD3751 (to SL and DRP), a grant-in-aid from the Spanish Society of Nephrology (Fundación Senefro 2017 to SL), and Fundación Renal "Iñigo Alvarez de Toledo" (to SL), all from Spain. The Centro de Biología Molecular "Severo Ochoa" (CBMSO) receives institutional support from Fundación "Ramón Areces." SL was supported by the "Salvador de Madariaga" grant PRX15/00119 from the Spanish Ministry of Educación, Cultura y Deporte, for a 4-month stay at the Yale lab. VM was supported by a predoctoral fellowship of the FPI Program (BES2013-065986) from MINECO. We are grateful to the laboratories of Jorgina Satrustegui and José Manuel Cuezva at the CBMSO for sharing the Seahorse equipment.

Address correspondence to: Carlos Fernández-Hernando, 10 Amistad Street, Room 337C, New Haven, Connecticut 06520, USA. Phone: 203.737.4615; Email: carlos.fernandez@yale.edu. Or to: Santiago Lamas, Centro de Biología Molecular Severo Ochoa, Calle Nicolás Cabrera 1, Madrid 28049, Spain. Phone: 34.911964455; Email: slamas@cbm.csic.es.

1. Könner AC, Brüning JC. Selective insulin and leptin resistance in metabolic disorders. Cell Metab. 2012;16(2):144-152.

2. Woo KT, Choong HL, Wong KS, Tan HB, Chan CM. The contribution of chronic kidney disease to the global burden of major noncommunicable diseases. Kidney Int. 2012;81(10):1044-1045.

3. Nath KA. Tubulointerstitial changes as a major determinant in the progression of renal damage. Am J Kidney Dis. 1992;20(1):1-17.

4. Duffield JS. Cellular and molecular mechanisms in kidney fibrosis. J Clin Invest. 2014;124(6):2299-2306.

5. Nieth H, Schollmeyer P. Substrate-utilization of the human kidney. Nature. 1966;209(5029):1244-1245.

6. Handschin C, Spiegelman BM. Peroxisome proliferator-activated receptor gamma coactivator 1 coactivators, energy homeostasis, and metabolism. Endocr Rev. 2006;27(7):728-735.

7. Reidy K, Kang HM, Hostetter T, Susztak K. Molecular mechanisms of diabetic kidney disease. J Clin Invest. 2014;124(6):2333-2340.

8. Tang C, Kanter JE, Bornfeldt KE, Leboeuf RC, Oram JF. Diabetes reduces the cholesterol exporter ABCA1 in mouse macrophages and kidneys. J Lipid Res. 2010;51(7):1719-1728.

9. Stadler K, Goldberg IJ, Susztak K. The evolving understanding of the contribution of lipid metabolism to diabetic kidney disease. Curr Diab Rep. 2015;15(7):40.

10. Huang S, et al. Jagged1/Notch2 controls kidney fibrosis via Tfam-mediated metabolic reprogramming. PLoS Biol. 2018;16(9):e2005233.

11. Kang HM, et al. Defective fatty acid oxidation in renal tubular epithelial cells has a key role in kidney fibrosis development. Nat Med. 2015;21(1):37-46.

12. Kruger C, et al. Proximal tubular cell-specific ablation of carnitine acetyltransferase causes tubular disease and secondary glomerulosclerosis. Diabetes. 2019;68(4):819-831.

13. Han SH, et al. PGC-1 $\alpha$ protects from Notch-induced kidney fibrosis development. J Am Soc Nephrol. 2017;28(11):3312-3322.

14. Simon N, Hertig A. alteration of fatty acid oxidation in tubular epithelial cells: from acute kidney injury to renal fibrogenesis. Front Med (Lausanne). 2015;2:52.

15. Breyer MD, Susztak K. The next generation of therapeutics for chronic kidney disease. Nat Rev Drug Discov. 2016;15(8):568-588.

16. Price NL, et al. Specific disruption of Abca1 targeting largely mimics the effects of miR-33 knockout on macrophage cholesterol efflux and atherosclerotic plaque development. Circ Res. 2019;124(6):874-880.

17. Gomez IG, et al. Anti-microRNA-21 oligonucleotides prevent Alport nephropathy progression by stimulating metabolic pathways. J Clin Invest. 2015;125(1):141-156.

18. Gomez IG, Nakagawa N, Duffield JS. MicroRNAs as novel therapeutic targets to treat kidney injury and fibrosis. Am J Physiol Renal Physiol. 2016;310(10):F931-F944.

19. Chung AC, Lan HY. MicroRNAs in renal fibrosis. Front Physiol. 2015;6:50.

20. Denby L, et al. MicroRNA-214 antagonism protects against renal fibrosis. J Am Soc Nephrol. 2014;25(1):65-80.

21. Denby L, et al. miR-21 and miR-214 are consistently modulated during renal injury in rodent models. Am J Pathol. 2011;179(2):661-672.

22. Chau BN, et al. MicroRNA-21 promotes fibrosis of the kidney by silencing metabolic pathways. Sci Transl Med. 2012;4(121):121ra18.

23. Wang B, et al. Suppression of microRNA-29 expression by TGF- $\beta 1$ promotes collagen expression and renal fibrosis. $J$ Am Soc Nephrol. 2012;23(2):252-265.

24. Najafi-Shoushtari SH, et al. MicroRNA-33 and the SREBP host genes cooperate to control cholesterol homeostasis. Science. 2010;328(5985):1566-1569.

25. Rayner KJ, et al. MiR-33 contributes to the regulation of cholesterol homeostasis. Science. 2010;328(5985):1570-1573

26. Dávalos A, et al. miR-33a/b contribute to the regulation of fatty acid metabolism and insulin signaling. Proc Natl Acad Sci USA. 2011;108(22):9232-9237.

27. Gerin I, et al. Expression of miR-33 from an SREBP2 intron inhibits cholesterol export and fatty acid oxidation. J Biol Chem. 2010;285(44):33652-33661.

28. Cao M, Bai L, Wang D, Zhai Q, Li Y, Hai J, Wang W. miRNA-33 expression and its mechanism in patients and model rats with 
type 2 diabetic nephropathy. Int J Clin Exp Med. 2018;11(3):1661-1668.

29. Ouimet M, et al. MicroRNA-33-dependent regulation of macrophage metabolism directs immune cell polarization in atherosclerosis. J Clin Invest. 2015;125(12):4334-4348

30. D'Agati VD, et al. Obesity-related glomerulopathy: clinical and pathologic characteristics and pathogenesis. Nat Rev Nephrol. 2016;12(8):453-471.

31. Goedeke L, et al. A regulatory role for microRNA 33* in controlling lipid metabolism gene expression. Mol Cell Biol. 2013;33(11):2339-2352.

32. Zhang X, Price NL, Fernández-Hernando C. Non-coding RNAs in lipid metabolism. Vascul Pharmacol. 2019;114:93-102.

33. Goedeke L, et al. Long-term therapeutic silencing of miR-33 increases circulating triglyceride levels and hepatic lipid accumulation in mice. EMBO Mol Med. 2014;6(9):1133-1141.

34. Price NL, et al. SREBP-1c/MicroRNA 33b genomic loci control adipocyte differentiation. Mol Cell Biol. 2016;36(7):1180-1193.

35. Price NL, et al. Genetic dissection of the impact of miR-33a and miR-33b during the progression of atherosclerosis. Cell Rep. 2017;21(5):1317-1330

36. Cheng CJ, et al. MicroRNA silencing for cancer therapy targeted to the tumour microenvironment. Nature. 2015;518(7537):107-110

37. Arruda JA, Kurtzman NA. Relationship of renal sodium and water transport to hydrogen ion secretion. Annu Rev Physiol. 1978;40:43-66.

38. Andreev OA, et al. Mechanism and uses of a membrane peptide that targets tumors and other acidic tissues in vivo. Proc Natl Acad Sci USA. 2007;104(19):7893-7898.

39. Pálsson R, Patel UD. Cardiovascular complications of diabetic kidney disease. Adv Chronic Kidney Dis. 2014;21(3):273-280.

40. Chen HC, Guh JY, Chang JM, Hsieh MC, Shin SJ, Lai YH. Role of lipid control in diabetic nephropathy. Kidney Int Suppl. 2005;(94):S60-2.

41. Price NL, Ramírez CM, Fernández-Hernando C. Relevance of microRNA in metabolic diseases. Crit Rev Clin Lab Sci. 2014;51(6):305-320

42. Imig JD, Ryan MJ. Immune and inflammatory role in renal disease. Compr Physiol. 2013;3(2):957-976.

43. Wada T, Sakai N, Sakai Y, Matsushima K, Kaneko S, Furuichi K. Involvement of bone-marrow-derived cells in kidney fibrosis. Clin Exp Nephrol. 2011;15(1):8-13.

44. Guiteras R, Flaquer M, Cruzado JM. Macrophage in chronic kidney disease. Clin Kidney J. 2016;9(6):765-771.

45. Grande MT, et al. Snaill-induced partial epithelial-to-mesenchymal transition drives renal fibrosis in mice and can be targeted to reverse established disease. Nat Med. 2015;21(9):989-997.

46. Lovisa S, et al. Epithelial-to-mesenchymal transition induces cell cycle arrest and parenchymal damage in renal fibrosis. Nat Med. 2015;21(9):998-1009.

47. Bataille A, et al. Increased fatty acid oxidation in differentiated proximal tubular cells surviving a reversible episode of acute kidney injury. Cell Physiol Biochem. 2018;47(4):1338-1351.

48. Scerbo D, et al. Kidney triglyceride accumulation in the fasted mouse is dependent upon serum free fatty acids. J Lipid Res 2017;58(6):1132-1142.

49. Yang X, et al. CD36 in chronic kidney disease: novel insights and therapeutic opportunities. Nat Rev Nephrol. 2017;13(12):769-781.

50. Liu L, et al. DGAT1 expression increases heart triglyceride content but ameliorates lipotoxicity. J Biol Chem. 2009;284(52):36312-36323.

51. Li S, et al. Proximal tubule PPAR $\alpha$ attenuates renal fibrosis and inflammation caused by unilateral ureteral obstruction. Am $J$ Physiol Renal Physiol. 2013;305(5):F618-F627.

52. Tran M, et al. PGC-1 $\alpha$ promotes recovery after acute kidney injury during systemic inflammation in mice. J Clin Invest. 2011;121(10):4003-4014.

53. Chung KW, Lee EK, Lee MK, Oh GT, Yu BP, Chung HY. Impairment of PPAR $\alpha$ and the fatty acid oxidation pathway aggravates renal fibrosis during aging. J Am Soc Nephrol. 2018;29(4):1223-1237.

54. Idrovo JP, Yang WL, Nicastro J, Coppa GF, Wang P. Stimulation of carnitine palmitoyltransferase 1 improves renal function and attenuates tissue damage after ischemia/reperfusion. J Surg Res. 2012;177(1):157-164.

55. Vettori S, Gay S, Distler O. Role of microRNAs in fibrosis. Open Rheumatol J. 2012;6:130-139.

56. Lv W, et al. Therapeutic potential of microRNAs for the treatment of renal fibrosis and CKD. Physiol Genomics. 2018;50(1):20-34

57. Tomita K, et al. Free cholesterol accumulation in hepatic stellate cells: mechanism of liver fibrosis aggravation in nonalcoholic steatohepatitis in mice. Hepatology. 2014;59(1):154-169.

58. Nishiga M, et al. MicroRNA-33 controls adaptive fibrotic response in the remodeling heart by preserving lipid raft cholesterol Circ Res. 2017;120(5):835-847.

59. Zhang X, Fernández-Hernando C. miR-33 regulation of adaptive fibrotic response in cardiac remodeling. Circ Res. 2017;120(5):753-755.

60. Chevalier RL, Forbes MS, Thornhill BA. Ureteral obstruction as a model of renal interstitial fibrosis and obstructive nephropathy. Kidney Int. 2009;75(11):1145-1152.

61. Fink M, Henry M, Tange JD. Experimental folic acid nephropathy. Pathology. 1987;19(2):143-149.

62. Miguel V, Busnadiego O, Fierro-Fernández M, Lamas S. Protective role for miR-9-5p in the fibrogenic transformation of human dermal fibroblasts. Fibrogenesis Tissue Repair. 2016;9:7.

63. Ding W, Yousefi K, Shehadeh LA. Isolation, characterization, and high throughput extracellular flux analysis of mouse primary renal tubular epithelial cells. J Vis Exp. 2018;(136):57718.

64. Dranka BP, et al. Assessing bioenergetic function in response to oxidative stress by metabolic profiling. Free Radic Biol Med. 2011;51(9):1621-1635.

65. Bligh EG, Dyer WJ. A rapid method of total lipid extraction and purification. Can J Biochem Physiol. 1959;37(8):911-917.

66. Huynh FK, Green MF, Koves TR, Hirschey MD. Measurement of fatty acid oxidation rates in animal tissues and cell lines. Meth Enzymol. 2014;542:391-405.

67. Kusaba T, Lalli M, Kramann R, Kobayashi A, Humphreys BD. Differentiated kidney epithelial cells repair injured proximal tubule. Proc Natl Acad Sci USA. 2014;111(4):1527-1532. 